\title{
Stacks of cyclic covers of projective spaces
}

\author{
Alessandro Arsie and Angelo Vistoli
}

\begin{abstract}
We define stacks of uniform cyclic covers of Brauer-Severi schemes, proving that they can be realized as quotient stacks of open subsets of representations, and compute the Picard group for the open substacks parametrizing smooth uniform cyclic covers. Moreover, we give an analogous description for stacks parametrizing triple cyclic covers of Brauer-Severi schemes of rank 1 that are not necessarily uniform, and give a presentation of the Picard group of the substacks corresponding to smooth triple cyclic covers.
\end{abstract}

\section{Introduction}

In [Vis98], the second author described the stack of $\mathcal{M}_{2}$ of smooth curves of genus 2 as the quotient stack of an open subscheme of a representation of $\mathrm{GL}_{2}$, and used this description to compute its integral Chow ring. In particular, he reproved the known result that its Picard group is cyclic of order 10. The key point for the existence of such a presentation for $\mathcal{M}_{2}$ is the fact that any smooth curve of genus 2 is hyperelliptic.

In this work we define a much wider class of stacks, parametrizing families of uniform cyclic cover of projective spaces that can be realized as quotient stacks of an open subset of a representation. Special cases are the stack $\mathcal{M}_{2}$, the stacks $\mathcal{H}_{g}$ parametrizing hyperelliptic curves of genus $g$ and also the stack parametrizing K3 surfaces expressed as double covers of $\mathbb{P}^{2}$ ramified along a smooth sextic (up to an automorphism of $\mathbb{P}^{2}$ ). Again, the key idea is that for the objects involved in families of uniform cyclic covers, one has a concrete description in terms of polynomials and equations, so that the corresponding stack is obtained as a quotient stack of an affine space parametrizing the corresponding polynomials, modulo the action of the relevant group.

The paper is organized as follows. In $\S 2$ we give the main definitions and constructions for uniform cyclic covers of a scheme (these are essentially what were known as simple cyclic covers, see [Cat84]). A detailed analysis of these and other types of covers can be found in [Par91]. Moreover, we set up the general categorical framework for uniform cyclic covers over a fixed scheme.

In $\S 3$ we restrict our analysis to uniform cyclic covers of families of projective spaces, i.e. BrauerSeveri schemes. We introduce our main object of interest, the fibered categories $\mathcal{H}(n, r, d)$ that parametrize families of uniform cyclic covers over Brauer-Severi schemes.

In $\S 4$ we describe $\mathcal{H}(n, r, d)$ and $\mathcal{H}_{\mathrm{sm}}(n, r, d)$ (the open substack corresponding to smooth uniform cyclic covers) as quotient stacks. We also suggest a natural compactification of $\mathcal{H}_{\mathrm{sm}}(n, r, d)$ via Kirwan's procedure in $\S 4$.

$\S 5$ is dedicated to the computation of the integral Picard group of the $\operatorname{stack} \mathcal{H}_{\mathrm{sm}}(n, r, d)$; we show that is it cyclic of order $r(r d-1)^{n} \operatorname{gcd}(d, n+1)$. As a corollary, we immediately get that the

Received 23 January 2003, accepted in final form 9 April 2003. 2000 Mathematics Subject Classification 14A20, 14C22, 14D20.

Keywords: algebraic stacks, Brauer-Severi schemes, equivariant Chow rings, Picard groups, quotient stacks, uniform cyclic covers.

Both authors partially supported by the University of Bologna, funds for selected research topics.

This journal is (C) Foundation Compositio Mathematica 2004. 


\section{A. Arsie And A. Vistoli}

Picard group of the stack $\mathcal{H}_{g}$ of hyperelliptic curves of genus $g$ is cyclic of order $2(2 g+1)$ if $g$ is even, and $4(2 g+1)$ if $g$ is odd.

Finally, in $\S 6$ we define and study the stacks $\mathcal{H}\left(1,3 ; d_{1}, d_{2}\right)$ of cyclic triple (not necessarily uniform) covers of the projective line and its open substack $\mathcal{H}_{\mathrm{sm}}\left(1,3 ; d_{1}, d_{2}\right)$ corresponding to smooth covers. We prove that this stack can also be represented as a quotient stack, and we give a presentation of its Picard group.

\section{Uniform cyclic covers of a scheme}

Fix a positive integer $r$; we will denote by $\boldsymbol{\mu}_{r}=\boldsymbol{\mu}_{r, \mathbb{Z}}$ the group scheme of the $r$ th roots of 1 over $\operatorname{Spec} \mathbb{Z}$.

DEFINITION 2.1. Let $Y$ be a scheme. A uniform cyclic cover of degree $r$ of $Y$ consists of a morphism of schemes $f: X \rightarrow Y$ together with an action of the group scheme $\boldsymbol{\mu}_{r}$ on $X$, such that for each point $q$ of $Y$, there is an affine neighborhood $V=\operatorname{Spec} R$ of $q$ in $Y$, together with an element $h \in R$ that is not a zero divisor, and an isomorphism of $V$-schemes $f^{-1}(V) \simeq \operatorname{Spec} R[x] /\left(x^{r}-h\right)$ which is $\boldsymbol{\mu}_{r}$-equivariant, when the right-hand side is given the obvious actions.

These coverings should be properly called dual cyclic, rather than cyclic, as $\boldsymbol{\mu}_{r}$ is Cartier dual to the constant group scheme $\mathbb{Z} / r \mathbb{Z}$; however, we avoid this so as not to make the terminology unduly heavy. In literature, they are also known as simple cyclic covers.

If $X \rightarrow Y$ is a uniform cyclic cover of degree $r$, then $Y=X / \boldsymbol{\mu}_{r}$; so, in fact, $Y$ is determined by the action of $\boldsymbol{\mu}_{r}$ on $X$.

Uniform cyclic covers of a scheme $Y$ form a category, that we denote by $\mathcal{H}(Y, r)$. The arrows are $\boldsymbol{\mu}_{r}$-equivariant isomorphisms of schemes over $Y$; all the arrows are invertible, so this category is a groupoid.

There is a very well-known description of uniform cyclic covers, as follows. If $f: X \rightarrow Y$ is a uniform cyclic cover, the sheaf of $\mathcal{O}_{Y}$-algebras $f_{*} \mathcal{O}_{X}$ admits an action of $\boldsymbol{\mu}_{r}$, hence there is a direct sum decomposition

$$
f_{*} \mathcal{O}_{X}=\mathcal{L}_{0} \oplus \mathcal{L}_{1} \oplus \cdots \oplus \mathcal{L}_{r-1},
$$

where $\mathcal{L}_{i}$ is the subsheaf of $f_{*} \mathcal{O}_{X}$ of sections $s$ where the action of $\boldsymbol{\mu}_{r}$ is described by the rule $(t, s) \mapsto t^{i} s$. The multiplication is $\boldsymbol{\mu}_{r}$ equivariant; therefore, for each $i=0, \ldots, r-1$ there is an induced homomorphism $\mathcal{L}_{1}^{\otimes i} \rightarrow \mathcal{L}_{i}$, and also $\mathcal{L}_{1}^{\otimes r} \rightarrow \mathcal{L}_{0}$. The local description of the morphism $X \rightarrow Y$ shows that the following facts are true.

a) Each $\mathcal{L}_{i}$ is an invertible sheaf on $Y$.

b) $\mathcal{L}_{0}=\mathcal{O}_{Y}$

c) For each $i=0, \ldots, r-1$, the homomorphism $\mathcal{L}_{1}^{\otimes i} \rightarrow \mathcal{L}_{i}$ is an isomorphism.

d) The homomorphism $\mathcal{L}_{1}^{\otimes r} \rightarrow \mathcal{O}_{Y}$ is injective.

The image of $\mathcal{L}_{1}^{\otimes r}$ in $\mathcal{O}_{Y}$ is the sheaf of ideals of a Cartier divisor on $Y$, which we denote by $\Delta_{f}$ or $\Delta_{X / Y}$ and call the branch divisor of the uniform cyclic cover. If $V=\operatorname{Spec} R$ is an open affine subset of $Y$, such that $f^{-1}(V) \simeq \operatorname{Spec} R[x] /\left(x^{r}-h\right)$ as in the definition, then the restriction of $\Delta_{f}$ to $V$ is the divisor of $h$.

Conversely, assume that we are given a scheme $Y$ with an invertible sheaf $\mathcal{L}$, together with an injective homomorphism $\phi: \mathcal{L}^{\otimes r} \rightarrow \mathcal{O}_{Y}$. We can give the sheaf of $\mathcal{O}_{Y}$-modules

$$
\mathcal{O}_{Y} \oplus \mathcal{L} \oplus \mathcal{L}^{\otimes 2} \oplus \cdots \oplus \mathcal{L}^{\otimes(r-1)}
$$




\section{StACKS OF CYCLIC COVERS OF PROJECTIVE SPACES}

a structure of $(\mathbb{Z} / r \mathbb{Z})$-graded algebra, by defining the product of an element $s \in \mathcal{L}^{\otimes i}$ and $t \in \mathcal{L}^{\otimes j}$ as

$$
s \otimes t \in \mathcal{L}^{\otimes(i+j)}
$$

if $i+j<r$, and as

$$
\phi \otimes \operatorname{id}(s \otimes t) \in \mathcal{L}^{\otimes(i+j-r)}
$$

if $i+j \geqslant r$, where

$$
\phi \otimes \mathrm{id}: \mathcal{L}^{\otimes(i+j)} \rightarrow \mathcal{O}_{Y} \otimes \mathcal{L}^{\otimes(i+j-r)}=\mathcal{L}^{\otimes(i+j-r)}
$$

is the obvious homomorphism. Consider the relative spectrum $X$ of this sheaf of algebras: the $\mathbb{Z} / r \mathbb{Z}$ grading yields an action of $\boldsymbol{\mu}_{r}$ over $X$, and it is immediate to verify that, in fact, $X \rightarrow Y$ is a uniform cyclic cover.

This analysis leads to the following conclusion. Define a category $\mathcal{H}^{\prime}(Y, r)$, whose objects $(\mathcal{L}, \phi)$ are invertible sheaves $\mathcal{L}$ on $Y$, together with an injective homomorphism of $\mathcal{O}(Y)$-modules $\phi: \mathcal{L}^{\otimes r} \rightarrow$ $\mathcal{O}_{Y}$. The arrows $\alpha:(\mathcal{L}, \phi) \rightarrow(\mathcal{M}, \psi)$ are isomorphisms of invertible sheaves $\alpha: \mathcal{L} \simeq \mathcal{M}$, making the diagram

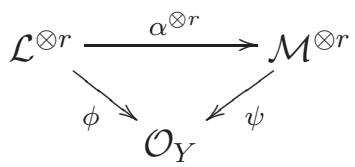

commutative.

Proposition 2.2. There is an equivalence of categories between the category $\mathcal{H}(Y, r)$ and the category $\mathcal{H}^{\prime}(Y, r)$.

Given a uniform cyclic cover $f: X \rightarrow Y$, the pullback of $\Delta_{f}$ to $X$ is a Cartier divisor, which is of the form $r D_{f}$, where $D_{f}$ is a Cartier divisor on $X$, whose sheaf of ideals is the pullback $f^{*} \mathcal{L}$, where $\mathcal{L}$ is the invertible sheaf associated with $f: X \rightarrow Y$. The restriction $D_{f} \rightarrow \Delta_{f}$ is an isomorphism.

There is a problem with defining pullbacks of uniform cyclic covers: if $f: X \rightarrow Y$ is a uniform cyclic cover and $Y^{\prime} \rightarrow Y$ a morphism of schemes, the pullback $X^{\prime} \stackrel{\text { def }}{=} Y^{\prime} \times_{Y} X$ acquires natural actions of $\boldsymbol{\mu}_{r}$, but the projection $f^{\prime}: X^{\prime} \rightarrow Y^{\prime}$ is a uniform cyclic cover if and only if the pullback of the branch divisor $\Delta_{f}$ to $Y^{\prime}$ is still a Cartier divisor. This problem does not arise in a relative context, which is what we are interested in.

Definition 2.3. Let $Y \rightarrow S$ be a morphism of schemes. A relative uniform cyclic cover $f: X \rightarrow Y$ is a uniform cyclic cover, such that the branch divisor $\Delta_{f}$ is flat over $S$.

By the local criterion of flatness, $f: X \rightarrow Y$ is a relative uniform cyclic cover if and only if $\Delta_{f}$ remains a Cartier divisor when restricted to any of the fiber of $Y \rightarrow S$.

The relative uniform cyclic covers over $Y \rightarrow S$ form a full subcategory of $\mathcal{H}(Y, r)$, denoted by $\mathcal{H}(Y / S, r)$

If $f: X \rightarrow Y$ is a relative uniform cyclic cover over $Y \rightarrow S$ and $S^{\prime} \rightarrow S$ is an arbitrary morphism of schemes, then the pullback of $\Delta_{f}$ to $S^{\prime} \times{ }_{S} Y$ is still a Cartier divisor, so the projection $S^{\prime} \times{ }_{S} X \rightarrow S^{\prime} \times{ }_{S} Y$ is a relative uniform cyclic cover.

Definition 2.4. A relative uniform cyclic cover $f: X \rightarrow Y$ over a morphism $Y \rightarrow S$ is smooth over $S$ if both $Y$ and the branch divisor $\Delta_{f}$ are smooth over $S$.

The proof of the following is straightforward.

Proposition 2.5. Let $Y \rightarrow S$ be a smooth morphism and $f: X \rightarrow Y$ be a relative uniform cyclic cover of degree $r$. Then $f$ is a smooth uniform cyclic cover over $S$ if and only if $X$ is smooth over $S$. 


\section{Uniform cyclic covers of projective spaces}

We are interested in relative uniform cyclic covers $f: X \rightarrow P$ of degree $r$, where $P \rightarrow S$ is a BrauerSeveri scheme. Given such a thing, consider the invertible sheaf $\mathcal{L}$ of sections of $f_{*} \mathcal{O}_{X}$ on which $\boldsymbol{\mu}_{r}$ acts via multiplication. The degree of such a invertible sheaf on the geometric fibers of $P \rightarrow S$ is a local invariant. We say that such a uniform cyclic cover has branch degree $d$ if the degree of $\mathcal{L}$ is $d$ on every fiber; the degree of the branch divisor is then equal to $r d$ (so perhaps this is not great terminology).

Fix three positive integers $n, r$ and $d$. We are interested in the category $\mathcal{H}(n, r, d)$, defined as follows.

An object $(X \stackrel{f}{\rightarrow} P \rightarrow S)$ of $\mathcal{H}(n, r, d)$ is a relative uniform cyclic cover $f: X \rightarrow P$ of degree $r$ and branch degree $d$, where $P \rightarrow S$ is a Brauer-Severi scheme of relative dimension $n$.

An arrow from $\left(X^{\prime} \stackrel{f^{\prime}}{\rightarrow} P^{\prime} \rightarrow S^{\prime}\right)$ to $(X \stackrel{f}{\rightarrow} P \rightarrow S)$ is a commutative diagram

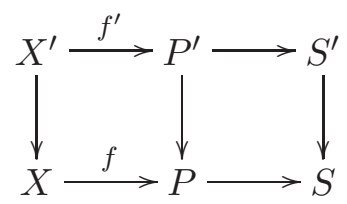

where both squares are Cartesian and the left-hand column is $\boldsymbol{\mu}_{r}$-equivariant.

We can reformulate the definition as follows.

Proposition 3.1. The category $\mathcal{H}(n, r, d)$ is equivalent to the category $\mathcal{H}^{\prime \prime}(n, r, d)$ defined as follows. The objects are flat and proper morphisms $X \rightarrow S$ of schemes, together with an action of $\boldsymbol{\mu}_{r}$ on $X$ leaving $X \rightarrow S$ invariant, satisfying the following condition: for any geometric point $s:$ Spec $\Omega \rightarrow X$, the action on $\boldsymbol{\mu}_{r}$ on the geometric fiber $X_{s}$ is faithful, the quotient $X_{s} / \boldsymbol{\mu}_{r}$ is isomorphic to $\mathbb{P}_{\operatorname{Spec} \Omega}^{n}$, and the projection $X_{s} \rightarrow X_{s} / \boldsymbol{\mu}_{r}$ makes $X_{s}$ into a uniform cyclic cover of $X_{s} / \boldsymbol{\mu}_{r}$, with degree $r$ and branch index $d$.

The arrows from $X^{\prime} \rightarrow S^{\prime}$ to $X \rightarrow S$ are commutative squares

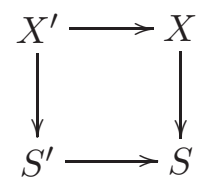

such that the top row is $\boldsymbol{\mu}_{r}$-equivariant.

Proof. Given an object $(X \rightarrow P \rightarrow S)$ of $\mathcal{H}(n, r, d)$, we have that the composition $X \rightarrow S$ gives an object of $\mathcal{H}^{\prime \prime}(n, r, d)$; this, together with the analogous construction for arrows, defines a functor $\mathcal{H}(n, r, d) \rightarrow \mathcal{H}^{\prime \prime}(n, r, d)$. To go in the other direction we need a lemma.

Lemma 3.2. If $X \rightarrow S$ is a morphism of schemes and there is given an action of $\boldsymbol{\mu}_{r}$ on $X$ leaving $X \rightarrow S$ invariant, then the formation of the quotient $X / \boldsymbol{\mu}_{r}$ commutes with base change on $S$. Furthermore, if $X$ is flat over $S$, so is $X / \boldsymbol{\mu}_{r}$.

Proof. Both parts of the statement are standard consequences of the fact that $\boldsymbol{\mu}_{r}$ is a diagonalizable group scheme over Spec $\mathbb{Z}$.

Suppose that $X \rightarrow S$ is an object of $\mathcal{H}^{\prime \prime}(n, r, d)$ and factor it as $X \rightarrow P \rightarrow S$, where $P=X / \boldsymbol{\mu}_{r}$. Obviously $P$ is proper over $S$. The lemma implies that it is also flat over $S$ and that the geometric fibers are projective spaces; hence, by a well-known theorem of Grothendieck, $P$ is a Brauer-Severi scheme over $S$. 


\section{StACKS OF CYCLIC COVERS OF PROJECTIVE SPACES}

Also, the restrictions of the projection morphism $f: X \rightarrow P$ over the points of $S$ is flat, so, by the local criterion of flatness, $f$ itself is flat. It is also finite, so $X$ can be thought of as the relative spectrum on $P$ of the locally free sheaf of algebras $f_{*} \mathcal{O}_{X}$, which we can decompose as

$$
f_{*} \mathcal{O}_{X}=\mathcal{L}_{0} \oplus \mathcal{L}_{1} \oplus \cdots \oplus \mathcal{L}_{r-1}
$$

using the action of $\boldsymbol{\mu}_{r}$. For each $i=0, \ldots, r-1$, the natural homomorphism $\mathcal{L}_{1}^{\otimes i} \rightarrow \mathcal{L}_{i}$ is an isomorphism on each geometric fiber, hence it is an isomorphism; furthermore, $\mathcal{L}_{1}^{\otimes r} \rightarrow \mathcal{O}_{P}$ is injective on the geometric fibers. This means that $f: X \rightarrow P$ is a uniform cyclic cover, hence $X \rightarrow P \rightarrow S$ is an object of $\mathcal{H}(n, r, d)$.

It is very easily checked that this extends naturally to a functor $\mathcal{H}^{\prime \prime}(n, r, d) \rightarrow \mathcal{H}(n, r, d)$ and this gives a quasi-inverse to the functor above. This concludes the proof of Proposition 3.1.

Remark 3.3. It is also convenient to define a fibered category $\mathcal{H}^{\prime}(n, r, d)$, in which an object over a scheme $S$ consists of the following set of data: a Brauer-Severi scheme $P \rightarrow S$; an invertible sheaf $\mathcal{L}$ on $P$, which restricts to a invertible sheaf of degree $-d$ on any geometric fiber; and an injection $i: \mathcal{L}^{\otimes r} \rightarrow \mathcal{O}_{P}$, which remains injective when restricted to any geometric fiber. The morphisms are defined in the obvious way. Clearly there is a morphism of fibered categories $p: \mathcal{H}(n, r, d) \rightarrow$ $\mathcal{H}^{\prime}(n, r, d)$ sending the object $(X \rightarrow P \rightarrow S)$ to the triple $\left(P \rightarrow S, \mathcal{L}, i: \mathcal{L}^{\otimes r} \rightarrow \mathcal{O}_{P}\right)$ and acting in the obvious way on morphisms. This correspondence is also an equivalence of the fibered category, as it is immediate to see, since $X$ can be recovered as $\underline{\operatorname{Spec}}_{\mathcal{O}_{P}}\left(\mathcal{O}_{P} \oplus \mathcal{L} \oplus \cdots \oplus \mathcal{L}^{\otimes r-1}\right)$.

We denote by $\mathcal{H}_{\mathrm{sm}}(n, r, d)$ the full subcategory of $\mathcal{H}(n, r, d)$ consisting of relative uniform cyclic covers $X \rightarrow P \rightarrow S$ which are smooth over the base.

There is a natural forgetful functor from $\mathcal{H}(n, r, d)$ to the category of schemes, sending $(X \stackrel{f}{\rightarrow}$ $P \rightarrow S)$ to $S$; this makes $\mathcal{H}(n, r, d)$ into a fiber category over the category of schemes and $\mathcal{H}_{\mathrm{sm}}(n, r, d)$ is a fibered subcategory.

From now on, if $R$ is a commutative ring, we write $\mathcal{H}(n, r, d)_{R}$ for the fiber product of $\mathcal{H}(n, r, d)$ with the category of schemes over $R$; the objects of $\mathcal{H}(n, r, d)_{R}$ are pairs $((X \rightarrow P \rightarrow S), S \rightarrow$ Spec $R$ ) consisting of an object of $\mathcal{H}(n, r, d)$ and of a morphism of schemes. The arrows are defined in the obvious way. There will be obvious variant of this notation, such as $\mathcal{H}_{\mathrm{sm}}(n, r, d)_{R}$ and $\mathcal{H}^{\prime}(n, r, d)_{R}$ (the category $\mathcal{H}^{\prime}(n, r, d)$ is defined above).

The category $\mathcal{H}_{\mathrm{sm}}(n, r, d)_{\mathbb{Z}[1 / r]}$ is fibered on the category of schemes over $\operatorname{Spec} \mathbb{Z}[1 / r]$. It has a simple description, using the equivalent description of $\mathcal{H}^{\prime}(n, r, d)$ given in Proposition 3.1.

Proposition 3.4. The fibered category $\mathcal{H}_{\mathrm{sm}}(n, r, d)_{\mathbb{Z}[1 / r]}$ is equivalent to the full subcategory of $\mathcal{H}^{\prime}(n, r, d)$ consisting of objects $X \rightarrow S$ which are smooth as morphisms of schemes and where $S$ is a scheme over Spec $\mathbb{Z}[1 / r]$.

The proof follows from Proposition 3.1 and Proposition 2.5.

Next are some examples of our construction.

Example 3.5.

a) For each $g \geqslant 2$, the fibered category $\mathcal{H}_{\mathrm{sm}}(1,2, g+1)_{\mathbb{Z}[1 / 2]}$ is a closed substack of the stack $\mathcal{M}_{g}$ of smooth curves of genus $g$, whose geometric points are the hyperelliptic curves. In particular, $\mathcal{H}_{\mathrm{sm}}(1,2,3)_{\mathbb{Z}[1 / 2]}$ coincides with $\mathcal{M}_{2}$.

b) We do not know if the category $\mathcal{H}_{\mathrm{sm}}(1,2,2)$ has appeared in the literature before. Its objects are smooth families $X \rightarrow S$ of curves of genus 1 over a scheme on Spec $\mathbb{Z}[1 / 2]$, together with an effective divisor $\Sigma \subseteq X$, such that the restriction $\Sigma \rightarrow S$ is étale of degree 4 and $\Sigma$ is invariant under the action of the 2-torsion part ${ }_{2} \operatorname{Pic}^{0}(X / S) \rightarrow S$ of the associated elliptic curve. 


\section{A. Arsie And A. Vistoli}

c) Consider the category $\mathcal{H}_{\mathrm{sm}}(2,2,3)$ of double covers of a projective plane, ramified over a smooth sextic curve. In characteristic different from 2, the resulting surfaces are K3 surfaces of a special and well-studied type.

Remark 3.6. More generally, we might be interested in flat morphisms $f: X \rightarrow P$, where $P \rightarrow S$ is a Brauer-Severi scheme, together with an action of $\boldsymbol{\mu}_{r}$ on $X$ leaving $f$ invariant, such that there exists an open subscheme $U$ of $P$, dense in every fiber of $P \rightarrow S$, such that, over $U$, the restriction of $f$ is a $\boldsymbol{\mu}_{r}$-torsor. Among these, uniform cyclic covers are special in two ways.

First of all, they are totally ramified (that is, the action of $\boldsymbol{\mu}_{r}$ is free outside of the fixed locus); of course, this is only a restriction when $r$ is not a prime.

Also, the action of $\boldsymbol{\mu}_{r}$ around a fixed point is of very restricted type; for example, if we are looking at a smooth uniform cyclic cover $f: X \rightarrow \mathbb{P}^{n}$ defined over $\mathbb{C}$, then the restriction $X^{\prime} \rightarrow \mathbb{P}^{n} \backslash \Delta_{f}$ is a Galois covering with group $\boldsymbol{\mu}_{r}$, whose restriction to a small loop $L \simeq \mathbb{S}^{1}$ around a smooth point of $\Delta_{f}$ corresponds to the canonical generator of $\mathrm{H}^{1}\left(L, \boldsymbol{\mu}_{r}\right)=\mathbb{Z} / r \mathbb{Z}$.

If $n>1$, we might consider this not to be a serious restriction; for example, if $r$ is a prime power, $f: X \rightarrow \mathbb{P}^{n}$ is a flat morphism defined over a field of characteristic prime to $r$ and there is an action of $\boldsymbol{\mu}_{r}$ on $X$ leaving $f$ invariant, such that generically $X$ is a torsor over $\mathbb{P}^{n}$ and $X$ is smooth over the base field, then it is not hard to show that we can make $f: X \rightarrow \mathbb{P}^{n}$ into a uniform cyclic cover by changing the action by an automorphism of $\boldsymbol{\mu}_{r}$; thus the resulting stack is a disjoint union of copies of $\mathcal{H}_{\mathrm{sm}}(n, r, d)$.

When $r$ is not a prime power, then this is not true anymore; however, we can still describe this stack as an open substack of products of stacks of type $\mathcal{H}_{\mathrm{sm}}\left(n, r_{i}, d_{i}\right)$.

Things are altogether different when $n=1$ and $r>2$; here the branch divisor will almost never be irreducible, and cyclic coverings of $\mathbb{P}^{1}$ that are not uniform are very common. We describe the situation for $\boldsymbol{\mu}_{3}$-covers in $\S 6$.

Remark 3.7. The stack $\mathcal{H}(n, r, d)$ itself is not particularly useful; the objects involved are highly unstable. We will be mostly interested in $\mathcal{H}_{\mathrm{sm}}(n, r, d)$; there is a natural compactification of it, via Kirwan's procedure, as explained in Remark 4.3.

\section{4. $\mathcal{H}(n, r, d)$ as a quotient stack}

For each triple $n, r$ and $d$, consider the space $\mathbb{A}(n, r d)$ of homogenous forms of degree $r d$ in $n+1$ indeterminates; we can think about $\mathbb{A}(n, r d)$ as the spectrum of the polynomial ring $\mathbb{Z}\left[a_{I}\right]$, where $a_{I}$ is an indeterminate and $I$ varies over the set of functions $I:\{0, \ldots, n\} \rightarrow \mathbb{N}$ with $\sum_{k} I(k)=r d$, so $\mathbb{A}(n, r d)$ is an affine space of dimension $\left(\begin{array}{c}r d+n \\ n\end{array}\right)$ over $\mathbb{Z}$.

We also write $\mathbb{P}(n, r d)$ for the projective space of lines in $\mathbb{A}(n, r d)$ (in this context, this convention seems more natural than Grothendieck's).

We denote by $\mathbb{A}_{0}(n, r d)$ the complement of the zero section Spec $\mathbb{Z} \hookrightarrow \mathbb{A}(n, r d)$ and by $\mathbb{A}_{\text {sm }}(n, r d)$ $\subseteq \mathbb{A}_{0}(n, r d)$ the open subscheme corresponding to smooth forms.

There is a natural action of $\mathrm{GL}_{n+1}=\mathrm{GL}_{n+1, \mathbb{Z}}$ on $\mathbb{A}(n, r d)$, defined, in functorial notation, by $A \cdot f(x)=f\left(A^{-1} x\right)$. The subgroup scheme $\boldsymbol{\mu}_{d} \subseteq \mathrm{GL}_{n+1}$, embedded by sending a $d$ th root of one $\alpha$ into the diagonal matrix $\alpha I_{n+1}$, acts trivially on $\mathbb{A}(n, r d)$, so this induces an action of the quotient $\mathrm{GL}_{n+1} / \boldsymbol{\mu}_{d}$ on $\mathbb{A}(n, r d)$, leaving the open subschemes $\mathbb{A}_{0}(n, r d)$ and $\mathbb{A}_{\mathrm{sm}}(n, r d)$ invariant.

Theorem 4.1. The fibered category $\mathcal{H}(n, r, d)$ is isomorphic to the quotient stack

$$
\left[\mathbb{A}_{0}(n, r d) /\left(\mathrm{GL}_{n+1} / \boldsymbol{\mu}_{d}\right)\right]
$$

by the action described above. 


\section{StaCks of CyCliC COVERS OF PROJECTIVE SPACES}

Furthermore, if $R$ is a commutative ring and $F \in \mathbb{A}_{0}(n, r d)(R)$ is a form of degree $r d$ whose coefficients generate the trivial ideal $R$, the branch divisor $\Delta_{f} \subseteq \mathbb{P}_{R}^{n}$ of the associated uniform cyclic cover $f: X \rightarrow \mathbb{P}_{R}^{n}$ is the hypersurface of $\mathbb{P}_{R}^{n}$ defined by $F$.

Proof. To prove the theorem, we identify $\mathcal{H}(n, r, d)$ with $\mathcal{H}^{\prime}(n, r, d)$, the fibered category of Remark 3.3.

Consider the auxiliary fibered category $\widetilde{\mathcal{H}}(n, r, d)$, whose objects over a base scheme $S$ are given as pairs consisting of an object $\left(P \rightarrow S, \mathcal{L}, i: \mathcal{L}^{\otimes r} \rightarrow \mathcal{O}_{P}\right)$ in $\mathcal{H}(n, r, d)(S)$, plus an isomorphism $\phi:(P, \mathcal{L}) \simeq\left(\mathbb{P}_{S}^{n}, \mathcal{O}(-d)\right)$ over $S$ (by this we mean the pair consisting of an isomorphism of $S$-schemes $\phi_{0}: P \simeq \mathbb{P}_{S}^{n}$, plus an isomorphism $\left.\phi_{1}: \mathcal{L} \simeq \phi_{0}^{*} \mathcal{O}(-d)\right)$. The arrows in $\widetilde{\mathcal{H}}(n, r, d)$ are arrows in $\mathcal{H}(n, r, d)$ preserving the isomorphisms $\phi$.

The obvious projection from $\widetilde{\mathcal{H}}(n, r, d)$ to the category of schemes makes it into a category fibered in groupoids. In fact, no object of $\widetilde{\mathcal{H}}(n, r, d)$ has a non-trivial automorphism mapping to identity in the category of schemes, so $\widetilde{\mathcal{H}}(n, r, d)$ is equivalent to a functor. We have a morphism of fibered categories from $\widetilde{\mathcal{H}}(n, r, d)$ to $\mathcal{H}^{\prime}(n, r, d)$ by forgetting the isomorphism $\phi$.

Let us define a base-preserving functor from $\widetilde{\mathcal{H}}(n, r, d)$ to $\mathbb{A}_{0}(n, r d)$. For any object of $\widetilde{\mathcal{H}}(n, r, d)(S)$ take the composition

$$
\phi \circ i \circ\left(\phi^{-1}\right)^{\otimes r}: \mathcal{O}_{\mathbb{P}_{S}^{n}}(-r d) \rightarrow \mathcal{O}_{\mathbb{P}_{S}^{n}},
$$

corresponding to a section of $\mathcal{O}_{\mathbb{P}_{S}^{n}}(r d)$ that does not vanish on any fiber of $\mathbb{P}_{S}^{n} \rightarrow S$; that is, to an element of $\mathbb{A}_{0}(n, r d)(S)$. There is also a base-preserving functor in the other direction, by sending a section $f \in \mathcal{O}_{\mathbb{P}_{S}^{n}}(r d)$, thought of as a homomorphism $f: \mathcal{O}_{\mathbb{P}_{S}^{n}}(-r d) \rightarrow \mathcal{O}_{\mathbb{P}_{S}^{n}}$, into the object

$$
\left(\mathbb{P}_{S}^{n} \rightarrow S, \mathcal{O}(-d), f: \mathcal{O}(-d)^{\otimes r} \rightarrow \mathcal{O}, \mathrm{id}:\left(\mathbb{P}_{S}^{n}, \mathcal{O}(-d)\right) \rightarrow\left(\mathbb{P}_{S}^{n}, \mathcal{O}(-d)\right)\right)
$$

of $\widetilde{\mathcal{H}}(n, r, d)(S)$. It is straightforward to check that this gives a quasi-inverse to the previous functor; so we get an equivalence of $\widetilde{\mathcal{H}}(n, r, d)$ with $\mathbb{A}_{0}(n, r d)$.

Now, for each integer $e$ consider the functor $\underline{\operatorname{Aut}}\left(\mathbb{P}_{\mathbb{Z}}^{n}, \mathcal{O}(e)\right)$ from schemes to groups sending each scheme $S$ into the group of automorphisms of the pair $\left(\mathbb{P}_{S}^{n}, \mathcal{O}(e)\right)$ over the identity on $S$. This is a sheaf in the fppf topology. Clearly, $\underline{\operatorname{Aut}}\left(\mathbb{P}_{\mathbb{Z}}^{n}, \mathcal{O}(1)\right)$ can be identified with $\mathrm{GL}_{n+1, \mathbb{Z}}$; an isomorphism of the pair $\left(\mathbb{P}_{S}^{n}, \mathcal{O}(1)\right)$, gives via $\pi: \mathbb{P}_{S}^{n} \rightarrow S$ an automorphism of $\pi_{*} \mathcal{O}(1)=\mathcal{O}_{S}^{n+1}$ as an $\mathcal{O}_{S}$-module and also works conversely. There is a natural homomorphism of sheaves of groups

$$
\underline{\operatorname{Aut}}\left(\mathbb{P}_{\mathbb{Z}}^{n}, \mathcal{O}(1)\right) \rightarrow \underline{\operatorname{Aut}}\left(\mathbb{P}_{\mathbb{Z}}^{n}, \mathcal{O}(e)\right)
$$

sending each automorphism $\left(\phi_{0}, \phi_{1}\right):\left(\mathbb{P}_{S}^{n}, \mathcal{O}(1)\right) \simeq\left(\mathbb{P}_{S}^{n}, \mathcal{O}(1)\right)$ into

$$
\left(\phi_{0}, \phi_{1}^{\otimes e}\right):\left(\mathbb{P}_{S}^{n}, \mathcal{O}(e)\right) \simeq\left(\mathbb{P}_{S}^{n}, \mathcal{O}(1)\right) .
$$

It is easy to check that this is a surjective homomorphism of fppf sheaves. If we identify $\underline{\operatorname{Aut}}\left(\mathbb{P}_{\mathbb{Z}}^{n}, \mathcal{O}(1)\right)$ with $\mathrm{GL}_{n+1, \mathbb{Z}}$, then the kernel of this homomorphism is the subgroup $\boldsymbol{\mu}_{|e|, \mathbb{Z}}$ embedded diagonally. So we get an isomorphism

$$
\underline{\operatorname{Aut}}\left(\mathbb{P}_{\mathbb{Z}}^{n}, \mathcal{O}(-d)\right) \simeq \mathrm{GL}_{n+1, \mathbb{Z}} / \boldsymbol{\mu}_{d, \mathbb{Z}} .
$$

There is a left action of $\underline{\operatorname{Aut}}\left(\mathbb{P}_{\mathbb{Z}}^{n}, \mathcal{O}(-d)\right)$ on $\widetilde{\mathcal{H}}(n, r, d)$; if

$$
\left(P \rightarrow S, \mathcal{L}, i: \mathcal{L}^{\otimes r} \rightarrow \mathcal{O}_{P}, \phi:(P, \mathcal{L}) \simeq\left(\mathbb{P}_{S}^{n}, \mathcal{O}(-d)\right)\right)
$$

is an object of $\widetilde{\mathcal{H}}(n, r, d)(S)$ and

$$
\alpha:\left(\mathbb{P}_{S}^{n}, \mathcal{O}(-d)\right) \simeq\left(\mathbb{P}_{S}^{n}, \mathcal{O}(-d)\right)
$$

is an element of $\underline{\operatorname{Aut}}\left(\mathbb{P}_{\mathbb{Z}}^{n}, \mathcal{O}(-d)\right)$, we associate with these the object

$$
\left(P \rightarrow S, \mathcal{L}, i: \mathcal{L}^{\otimes r} \rightarrow \mathcal{O}_{P}, \alpha \circ \phi:(P, \mathcal{L}) \simeq\left(\mathbb{P}_{S}^{n}, \mathcal{O}(-d)\right)\right)
$$




\section{A. Arsie And A. Vistoli}

Furthermore, given an invertible sheaf $\mathcal{L}$ on $P \rightarrow S$ whose degree is $-d$ on every geometric fiber, there is an fppf covering $S^{\prime} \rightarrow S$, such that the pullback of the pair $(P, \mathcal{L})$ to $S^{\prime}$ is isomorphic to $\left(\mathbb{P}_{S^{\prime}}^{n}, \mathcal{O}(-d)\right)$; this fact, plus descent theory, implies that the forgetful morphism $\widetilde{\mathcal{H}}(n, r, d) \rightarrow$ $\mathcal{H}(n, r, d)$ makes $\widetilde{\mathcal{H}}(n, r, d)$ into a principal bundle with group $\underline{\operatorname{Aut}}\left(\mathbb{P}_{\mathbb{Z}}^{n}, \mathcal{O}(-d)\right)=\mathrm{GL}_{n+1, \mathbb{Z}} / \boldsymbol{\mu}_{r, \mathbb{Z}}$.

If we identify $\widetilde{\mathcal{H}}(n, r, d)$ with $\mathbb{A}_{0}(n, r d)$, we obtain that $\mathcal{H}(n, r, d)$ is isomorphic to the quotient stack $\left[\mathbb{A}_{0}(n, r d) /\left(\mathrm{GL}_{n+1, \mathbb{Z}} / \boldsymbol{\mu}_{r, \mathbb{Z}}\right)\right]$. Now we only have to identify the action explicitly. However, from the description above it is easy to check that $\mathrm{GL}_{n+1}=\underline{\mathrm{Aut}}\left(\mathbb{P}_{\mathbb{Z}}^{n}, \mathcal{O}(1)\right)$ acts by the usual action $(f \cdot A)(x)=f\left(A^{-1} x\right)$, so the action of its quotient is that described above.

The last statement follows easily by construction.

The following corollary is a direct application of Theorem 4.1.

COROLlary 4.2. The fibered category $\mathcal{H}_{\mathrm{sm}}(n, r, d)$ is equivalent to the quotient stack

$$
\left[\mathbb{A}_{\mathrm{sm}}(n, r d) /\left(\mathrm{GL}_{n+1} / \boldsymbol{\mu}_{d}\right)\right]
$$

by the action described above.

In particular, $\mathcal{H}(n, r, d)$ is an irreducible smooth algebraic stack of finite type over Spec $\mathbb{Z}$, of relative dimension

$$
\left(\begin{array}{c}
r d+n \\
n
\end{array}\right)-(n+1)^{2}
$$

and $\mathcal{H}_{\mathrm{sm}}(n, r, d)$ is an open substack, hence it also smooth of the same dimension. So, for example, the dimension of the stack of hyperelliptic curves $\mathcal{H}_{\mathrm{sm}}(1,2, g+1)$ is $2 g-1$, as it should be, and the dimension of the stack of $\mathrm{K} 3$ surfaces $\mathcal{H}_{\mathrm{sm}}(2,2,3)$ is 19 .

The fact that hypersurfaces of degree at least three are stable for the action of $\mathrm{SL}_{n+1}$ implies that when $d>1$, the diagonal of $\mathcal{H}_{\mathrm{sm}}(n, r, d)$ is finite and its moduli space is quasiprojective over Spec $\mathbb{Z}$. Also, again for $d>1$, the restriction of $\mathcal{H}_{\mathrm{sm}}(n, r, d)_{\text {Spec } \mathbb{Z}[1 / r d]}$ is a Deligne-Mumford stack over $\operatorname{Spec} \mathbb{Z}[1 / r d]$.

Remark 4.3. Assume that $d$ is at least three. Then, if we look at the natural action of $\mathrm{SL}_{n+1}$ on the projectivization $\mathbb{P}(n, r d)$ of $\mathbb{A}(n, r d)$, the points corresponding to smooth hypersurfaces are stable. This implies that we can apply Kirwan's procedure (see [Kir85]) to get a canonical $\mathrm{GL}_{n+1} / \boldsymbol{\mu}_{d}$-equivariant morphism $K(n, r d) \rightarrow \mathbb{A}_{0}(n, r d)$ which is an isomorphism over $\mathbb{A}_{\mathrm{sm}}(n, r d)$, such that the action of $\mathrm{GL}_{n+1} / \boldsymbol{\mu}_{d}$ is proper and the geometric quotient $K(n, r d) /\left(\mathrm{GL}_{n+1} / \boldsymbol{\mu}_{d}\right)$ is a projective scheme over Spec $\mathbb{Z}$. The quotient stack

$$
\overline{\mathcal{H}}(n, r, d)=\left[K(n, r d) /\left(\mathrm{GL}_{n+1} / \boldsymbol{\mu}_{d}\right)\right]
$$

is an Artin stack with finite diagonal and projective moduli space, yielding a canonical compactification of $\mathcal{H}_{\mathrm{sm}}(n, r, d)$; this seems like a much more natural object than $\mathcal{H}(n, r, d)$.

We could try to investigate the stacks $\overline{\mathcal{H}}(n, r, d)$ and, in particular, describe their objects directly. This seems very complicated in dimensions higher than two, but at least for $n=1$ the problem should be approachable. If we exclude characteristic 2 then $\mathcal{H}(1,2,3)$ is the stack $\mathcal{M}_{2}$ of smooth curves of genus 2 and we can check that $\overline{\mathcal{H}}(1,2,3)$ is not isomorphic to the stack $\overline{\mathcal{M}}_{2}$ of stable curves of genus 2, although it would seem that it gives the same moduli space. However, in the next case, $\mathcal{H}(1,2,4)$ is the stack of smooth hyperelliptic curves of genus 3 and we can easily see that $\overline{\mathcal{H}}(1,2,4)$ does not coincide with the closure of $\mathcal{H}(1,2,4)$ inside $\overline{\mathcal{M}}_{3}$, not even at the level of moduli spaces; so the stack of hyperelliptic curves of fixed genus $g$ has two natural compactifications and, in general, they do not coincide. 


\section{StACKS OF CYCLIC COVERS OF PROJECTIVE SPACES}

It would be interesting to investigate these two compactifications and try to determine if they have any relations.

The group $\mathrm{GL}_{n+1} / \boldsymbol{\mu}_{d}$ appearing in the statement of the theorem can sometimes be written in a more familiar form. The following is straightforward.

\section{Proposition 4.4 .}

a) If $d \equiv 0(\bmod n+1)$, write $d=q(n+1)$. The homomorphism of group schemes over $\mathbb{Z}$

$$
\mathrm{GL}_{n+1} / \boldsymbol{\mu}_{d} \longrightarrow \mathbb{G}_{\mathrm{m}} \times \mathrm{PGL}_{n+1},
$$

defined by

$$
[A] \mapsto\left(\operatorname{det}(A)^{q},[A]\right),
$$

is an isomorphism.

b) If $d \equiv 1(\bmod n+1)$, write $d=q(n+1)+1$. The homomorphism of group schemes over $\mathbb{Z}$

$$
\mathrm{GL}_{n+1} / \boldsymbol{\mu}_{d} \rightarrow \mathrm{GL}_{n+1},
$$

defined by

$$
[A] \mapsto \operatorname{det}(A)^{q} A,
$$

is an isomorphism.

c) If $d \equiv-1(\bmod n+1)$, write $d=q(n+1)-1$. The homomorphism of group schemes over $\mathbb{Z}$

$$
\mathrm{GL}_{n+1} / \boldsymbol{\mu}_{d} \rightarrow \mathrm{GL}_{n+1},
$$

defined by

$$
[A] \mapsto \operatorname{det}(A)^{-q} A,
$$

is an isomorphism.

Remark 4.5. We can show that the group scheme $\mathrm{GL}_{n+1} / \boldsymbol{\mu}_{d}$ is isomorphic to $\mathrm{GL}_{n+1}$ if and only if $d \equiv \pm 1(\bmod n+1)$; on the other hand $\mathrm{GL}_{n+1} / \boldsymbol{\mu}_{d}$ is special (in the sense that every $\mathrm{GL}_{n+1} / \boldsymbol{\mu}_{d}$-torsor is locally trivial in the Zariski topology) if and only if $d$ is prime to $n+1$ (Zinovy Reichstein pointed this out to us). Experience teaches us that special groups are infinitely easier to handle than non-special ones; so, computing basic invariants of the spaces $\mathcal{H}(n, r, d)$ and $\mathcal{H}_{\mathrm{sm}}(n, r, d)$ (such as Chow rings and cohomology) should be much easier when $d$ is prime to $n+1$. For this purpose, it would be useful to gather information about the cohomology and the Chow ring of the classifying spaces of these groups.

If we rewrite the action of Theorem 4.1 via the isomorphisms of Proposition 4.4 we obtain the following.

Corollary 4.6.

a) If $d \equiv 0(\bmod n+1)$, write $d=q(n+1)$. Then $\mathcal{H}(n, r, d)$ is equivalent to the quotient stack

$$
\left[\mathbb{A}(n, r d) /\left(\mathbb{G}_{\mathrm{m}} \times \mathrm{PGL}_{n+1}\right)\right]
$$

by the action defined by the formula

$$
(\alpha,[A]) \cdot f(x)=\alpha^{-r} \operatorname{det}(A)^{r q} f\left(A^{-1} x\right) .
$$

b) If $d \equiv 1(\bmod n+1)$, write $d=q(n+1)+1$. Then $\mathcal{H}(n, r, d)$ is equivalent to the quotient stack

$$
\left[\mathbb{A}(n, r d) /\left(\mathrm{GL}_{n+1}\right)\right]
$$

by the action defined by the formula

$$
A \cdot f(x)=\operatorname{det}(A)^{r q} f\left(A^{-1} x\right) .
$$


A. Arsie And A. Vistoli

c) If $d \equiv-1(\bmod n+1)$, write $d=q(n+1)-1$. Then $\mathcal{H}(n, r, d)$ is equivalent to the quotient stack

$$
\left[\mathbb{A}(n, r d) /\left(\mathrm{GL}_{n+1}\right)\right]
$$

by the action defined by the formula

$$
A \cdot f(x)=\operatorname{det}(A)^{-r q} f\left(A^{-1} x\right) .
$$

In particular, we get the following description of the stack of hyperelliptic curves.

COROLlary 4.7. The stack $\mathcal{H}_{\mathrm{sm}}(1,2, g+1)$ of smooth hyperelliptic curves of genus $g$ is isomorphic to:

a) the quotient of $\mathbb{A}_{\mathrm{sm}}(1,2 g+2)$ by the action of $\mathrm{GL}_{2}$ defined by $A \cdot f(x)=\operatorname{det}(A)^{g} f\left(A^{-1} x\right)$ if $g$ is even; and

b) the quotient of $\mathbb{A}_{\mathrm{sm}}(1,2 g+2)$ by the action of $\mathbb{G}_{\mathrm{m}} \times \mathrm{PGL}_{2}$ defined by $(\alpha,[A]) \cdot f(x)=$ $\alpha^{-2} \operatorname{det}(A)^{g+1} f\left(A^{-1} x\right)$ if $g$ is odd.

When $g=2$, we recover the description of the stack $\mathcal{M}_{2}$ of smooth curves of genus 2 given in [Vis98]; the derivation here is much simpler, but the method of [Vis98] has some independent interest.

\section{Picard groups of stacks of smooth cyclic coverings}

We use the description of $\mathcal{H}_{\mathrm{sm}}(n, r, d)$ given in Corollary 4.2 to compute its Picard group, away from some bad characteristics.

Recall that if $\mathcal{X}$ is an algebraic stack over a scheme $S$, its Picard group is the group of isomorphism classes of invertible sheaves on $\mathcal{X}$, with the operation given as usual by tensor product. An invertible sheaf is a quasicoherent sheaf over $\mathcal{X}$, defined as in [LM00], which is locally free of rank 1 when restricted to an atlas.

The Picard group of the stack $\mathcal{M}_{1,1}$ of elliptic curves was first computed by Mumford in the legendary paper [Mum65], written before the notion of algebraic stack was introduced.

TheOREM 5.1. Let $R$ be a unique factorization domain such that the characteristic of its quotient field does not divide $2 r d$. Then the Picard group of the stack $\mathcal{H}_{\mathrm{sm}}(n, r, d)_{R}$ is cyclic, of order

$$
r(r d-1)^{n} \operatorname{gcd}(d, n+1) .
$$

Proof. First of all, it follows from the following lemma that we can assume that $R$ is a field.

Lemma 5.2. Let $\mathcal{X}$ be a flat regular algebraic stack of finite type over a unique factorization domain $R$ with quotient field $K$. Assume that the fibers of $\mathcal{X}$ over the closed points of $\operatorname{Spec} R$ are integral. Then the restriction homomorphism

$$
\operatorname{Pic} \mathcal{X} \longrightarrow \operatorname{Pic}\left(\operatorname{Spec} K \times_{\operatorname{Spec} R} \mathcal{X}\right)
$$

is an isomorphism.

Proof. The group of divisors Div $\mathcal{X}$ is the free abelian group generated by integral closed substacks of codimension one in $\mathcal{X}$. Effective divisors are defined in the usual fashion.

The group Div $\mathcal{X}$ can also be defined as follows: closed substacks of $\mathcal{X}$ that are local complete intersection of codimension one form a monoid with the cancellation property, the operation being defined by taking products of sheaves of ideals. It is the free abelian monoid on the set of integral closed substacks of codimension one in $\mathcal{X}$. The group Div $\mathcal{X}$ is the group of quotients of this monoid. 


\section{STACKS OF CYCLIC COVERS OF PROJECTIVE SPACES}

If $f: \mathcal{X}^{\prime} \rightarrow \mathcal{X}$ is a dominant morphism of noetherian regular algebraic stacks, any closed local complete intersection substack of $\mathcal{X}$ of codimension one pulls back to a closed local complete intersection substack of $\mathcal{X}^{\prime}$ of codimension one; this induces a group homomorphism $f^{*}: \operatorname{Div} \mathcal{X} \rightarrow \operatorname{Div} \mathcal{X}^{\prime}$.

If $\mathcal{D}$ is a divisor on $\mathcal{X}$, we can associate with it a divisor $\mathcal{D}_{U}$ for each smooth morphism $U \rightarrow \mathcal{X}$, where $U$ is a scheme, so that, given two smooth morphisms $U \rightarrow \mathcal{X}$ and $V \rightarrow \mathcal{X}$, the pullbacks of $\mathcal{D}_{U}$ and $\mathcal{D}_{V}$ to $U \times{ }_{\mathcal{X}} V$ coincide with $\mathcal{D}_{U \times \mathcal{X} V}$. This is done as follows: write $\mathcal{D}$ as $\mathcal{D}^{+}-\mathcal{D}^{-}$, where $\mathcal{D}^{+}$ and $\mathcal{D}^{-}$are effective and do not intersect in codimension one; they correspond to closed substacks of $\mathcal{X}$; they pull back to effective divisors $\mathcal{D}_{U}^{+}$and $\mathcal{D}_{U}^{-}$on $U$. We define $\mathcal{D}_{U}$ to be the difference $\mathcal{D}_{U}^{+}-\mathcal{D}_{U}^{-}$.

Conversely, if we are given a collection of divisors $D_{U}$ on $U$ for each smooth morphism $U \rightarrow \mathcal{X}$, where $U$ is a scheme, such that given two smooth morphisms $U \rightarrow \mathcal{X}$ and $V \rightarrow \mathcal{X}$ the pullbacks of $D_{U}$ and $D_{V}$ to $U \times{ }_{\mathcal{X}} V$ coincide with $D_{U \times \mathcal{X} V}$, there is a unique divisor $\mathcal{D}$ such that $\mathcal{D}_{U}=D_{U}$ for each smooth morphism $U \rightarrow \mathcal{X}$. If we write $D_{U}=D_{U}^{+}-D_{U}^{-}$, where $D_{U}^{+}$and $D_{U}^{-}$are effective and do not intersect in codimension one, for each pair of smooth morphisms $U \rightarrow \mathcal{X}$ and $V \rightarrow \mathcal{X}$ we have $D_{U \times \mathcal{X} V}^{+}=D_{U}^{+}$and $D_{U \times \mathcal{X} V}^{-}=D_{U}^{-}$; so $D_{U}^{+}$and $D_{U}^{-}$descend to closed substacks of codimension one, $\mathcal{D}^{+}$and $\mathcal{D}^{-}$of $\mathcal{X}$, whose ideals are locally generated by one element. We set $\mathcal{D}=\mathcal{D}^{+}-\mathcal{D}^{-}$.

If $\mathcal{D}$ is a divisor on $\mathcal{X}$, we can associate with it an invertible sheaf $\mathcal{O}(\mathcal{D})$ on $\mathcal{X}$, together with a non-vanishing section defined over the complement of the support of $\mathcal{D}$. Consider the invertible sheaf $\mathcal{O}\left(\mathcal{D}_{U}\right)$ defined over $U$ for each smooth morphism $U \rightarrow \mathcal{X}$. If $U \rightarrow \mathcal{X}$ and $V \rightarrow \mathcal{X}$ are smooth morphisms, there is a natural isomorphism of $\mathcal{O}\left(\mathcal{D}_{U \times \mathcal{X} V}\right)$ with the pullback of $\mathcal{O}\left(\mathcal{D}_{U}\right)$; these isomorphisms define the descent data for an invertible sheaf on $\mathcal{X}$ that we call $\mathcal{O}(\mathcal{D})$. On the complement of the support of $\mathcal{D}$, this invertible sheaf is canonically trivial.

This defines a group homomorphism Div $\mathcal{X} \rightarrow \operatorname{Pic} \mathcal{X}$. If $f: \mathcal{X}^{\prime} \rightarrow \mathcal{X}$ is a dominant morphism of noetherian regular algebraic stacks and $\mathcal{D}$ is a divisor on $\mathcal{X}$, then $\mathcal{O}\left(f^{*} \mathcal{D}\right)$ is canonically isomorphic to $f^{*} \mathcal{O}(\mathcal{D})$.

Conversely, if $\mathcal{L}$ is an invertible sheaf on $\mathcal{X}$ and $s$ is a nowhere vanishing section of $\mathcal{L}$ on an open dense substack $\mathcal{U}$, we can associate with it a divisor $\mathrm{Z}(s)$ on $\mathcal{X}$. If $\phi: U \rightarrow \mathcal{X}$ is a smooth morphism, we define $\mathrm{Z}(s)_{U}$ to be the divisor of the rational section $\phi^{*} s$ of the invertible sheaf $\phi^{*} \mathcal{L}$ on $U$.

We check immediately that $s$ extends to a nowhere vanishing function of the invertible sheaf $\mathcal{L} \otimes \mathcal{O}(-\mathrm{Z}(s))$; therefore, $\mathcal{L} \otimes \mathcal{O}(-\mathrm{Z}(s))$ is a trivial invertible sheaf and there is an isomorphism $\mathcal{L} \simeq \mathcal{O}(\mathrm{Z}(s))$.

Remark 5.3. In general, on a regular stack not all invertible sheaves come from divisors; those that do are precisely those possessing a rational section that does vanish on open dense substacks. For example, if $G$ is a finite group, the group of divisors on the associated classifying stack $\mathcal{B}_{\mathbb{C}} G$ is trivial, while the Picard group is the group of characters $G \rightarrow \mathbb{C}^{*}$ of $G$. In this case a rational section is an invariant and only the trivial character has non-zero invariants.

Now let us proceed with the proof of the lemma: set $\mathcal{X}_{K}=\operatorname{Spec} K \times_{\operatorname{Spec} R} \mathcal{X}$.

Let us show that the restriction homomorphism $\operatorname{Pic} \mathcal{X} \rightarrow \operatorname{Pic} \mathcal{X}_{K}$ is injective. Let $\mathcal{L}$ be an invertible sheaf on $\mathcal{X}$ whose restriction to $\mathcal{X}_{K}$ is trivial. Choose a nowhere vanishing section of the restriction of $\mathcal{L}$ to $\mathcal{X}_{K}$; this will extend to a nowhere vanishing section $s$ of $\mathcal{L}$ over some open substack $\mathcal{U}$ of $\mathcal{X}$ containing the fiber at infinity. Let $\mathcal{D}$ be the divisor on $\mathcal{X}$ defined by $s$; then, as we have seen, $\mathcal{L}$ is isomorphic to $\mathcal{O}(\mathcal{D})$. The support of $\mathcal{D}$ will be contained in a union of closed fibers of the morphism $\mathcal{X} \rightarrow \operatorname{Spec} R$; since these fibers are integral we see that $\mathcal{D}$ is the pullback of a divisor on $\operatorname{Spec} R$, so $\mathcal{L}$ is the pullback of an invertible sheaf on $\operatorname{Spec} R$. However, such an invertible sheaf is always trivial, because $R$ is a unique factorization domain.

To prove surjectivity, take an invertible sheaf $\mathcal{M}$ over $\mathcal{X}_{K}$ and consider the quasicoherent sheaf $j_{*} \mathcal{M}$ on $\mathcal{X}$, where $j: \mathcal{X}_{K} \rightarrow \mathcal{X}$ is the natural morphism. We claim that the natural homomorphism 


\section{A. Arsie And A. Vistoli}

$j^{*} j_{*} \mathcal{M} \rightarrow \mathcal{M}$ is an isomorphism. In fact, this is a local question in the smooth topology of $\mathcal{X}$, so we may assume that $\mathcal{X}$ is the spectrum of an $R$-algebra $A$ and then this follows from the fact that $\mathcal{X}_{K}$ is the spectrum of a localization of $A$.

It follows from [LM00, Proposition 15.4] that there exists a coherent subsheaf $\mathcal{F}$ of $j_{*} \mathcal{M}$ whose restriction to $\mathcal{X}_{K}$ coincides with $\mathcal{M}$. Then the double dual $\mathcal{F}^{\vee \vee}$ is a reflexive sheaf of rank one on a regular stack, so it is invertible and its restriction to $\mathcal{X}_{K}$ is isomorphic to $\mathcal{M}$. This completes the proof of the lemma.

So, assume that $R$ equals a field $k$. From the description of $\mathcal{H}_{\mathrm{sm}}(n, r, d)$ in Corollary 4.2 and from [EG98, Proposition 18] it follows that $\operatorname{Pic}\left(\mathcal{H}_{\mathrm{sm}}(n, r, d)\right)$ is equal to $\mathrm{A}_{\mathrm{GL}_{n+1} / \boldsymbol{\mu}_{d}}^{1}\left(\mathbb{A}_{\mathrm{sm}}(n, r d)\right)$, the codimension one component of the integral $\mathrm{GL}_{n+1} / \boldsymbol{\mu}_{d}$-equivariant Chow ring of $\mathbb{A}_{\mathrm{sm}}(n, r d)$.

Suppose that $G$ is an algebraic group over a field $k, V$ an $l$-dimensional representation of $G$ and $X$ an open invariant subscheme of $V$. If follows from [EG98] that the pullback $\mathrm{A}_{G}^{1} \stackrel{\text { def }}{=} \mathrm{A}_{G}^{1}(\mathrm{Spec} k) \rightarrow$ $\mathrm{A}_{G}^{1}(V)$ is an isomorphism. Indeed, $\mathrm{A}_{G}^{1}(\operatorname{Spec} k) \simeq \mathrm{A}_{-1}^{G}(\operatorname{Spec} k)$ and $\mathrm{A}_{G}^{1}(V) \simeq \mathrm{A}_{l-1}^{G}\left(V \times \times_{\operatorname{Spec} k} \operatorname{Spec} k\right)$ by [EG98, Proposition 4]; by [EG98, Theorem 1] we get $\mathrm{A}_{-1}^{G}(\operatorname{Spec} k) \simeq \operatorname{Pic}^{G}(\operatorname{Spec} k)$ and analogously for $\mathrm{A}_{l-1}^{G}\left(V \times \times_{\text {Spec } k} \operatorname{Spec} k\right)$. Finally, by [EG98, Lemma 2], if $\pi: V \times{ }_{\operatorname{Spec} k} \operatorname{Spec} k \rightarrow \operatorname{Spec} k$ is the second projection, then $\pi^{*}: \operatorname{Pic}^{G}(\operatorname{Spec} k) \rightarrow \operatorname{Pic}^{G}\left(V \times \times_{\operatorname{Spec} k} \operatorname{Spec} k\right)$ is an isomorphism and this yields the claim.

Again, $\mathrm{A}_{G}^{1}(\operatorname{Spec} k)$ is the equivariant Picard group for the trivial action of $G$ over Spec $k$; that is, is the group of characters $\widehat{G}$. Call $n$ the dimension of $V$. From the usual exact sequence

$$
\mathrm{A}_{n-1}^{G}(V \backslash X) \longrightarrow \mathrm{A}_{G}^{1}(V) \longrightarrow \mathrm{A}_{G}^{1}(X) \longrightarrow 0
$$

we see that $\mathrm{A}_{G}^{1}(X)$ is the quotient of $\widehat{G}$ by the subgroup generated by the classes of the components of $V \backslash X$ in codimension one. In our case, the group of characters $\widehat{\mathrm{GL}_{n+1}} / \boldsymbol{\mu}_{d}$ is infinite cyclic, while the locus $\Delta$ of singular forms is well known to be irreducible, so $\mathrm{A}_{G}^{1}\left(\mathbb{A}_{\mathrm{sm}}(n, r d)\right)$ is a cyclic group, of order equal to the index of the subgroup generated by the class of $\Delta$ in $\mathrm{A}^{1}(\mathbb{A}(n, r d))=\widehat{\mathrm{GL}_{n+1}} / \boldsymbol{\mu}_{d}$. To compute this index, first of all note that $\widehat{\mathrm{GL}_{n+1}} / \boldsymbol{\mu}_{d}$ injects inside $\widehat{\mathrm{GL}}_{n+1}$, which is generated by the determinant det: $\mathrm{GL}_{n+1} \rightarrow \mathbb{G}_{\mathrm{m}}$; since the intersection of $\boldsymbol{\mu}_{d}$ with the kernel of the determinant has order $\operatorname{gcd}(d, n+1)$, it follows that the index of $\widehat{\mathrm{GL}_{n+1}} / \boldsymbol{\mu}_{d}$ inside $\widehat{\mathrm{GL}}_{n+1}$ is $d / \operatorname{gcd}(d, n+1)$. In turn, if $\mathbb{G}_{\mathrm{m}} \hookrightarrow \mathrm{GL}_{n+1}$ is the usual embedding, $\widehat{\mathrm{GL}}_{n+1}$ has index $n+1$ in $\widehat{\mathbb{G}}_{\mathrm{m}}$; the composite homomorphism $\mathbb{G}_{\mathrm{m}} \rightarrow \mathrm{GL}_{n+1} / \boldsymbol{\mu}_{d}$ induces an embedding $\widehat{\mathrm{GL}_{n+1}} / \boldsymbol{\mu}_{d} \hookrightarrow \widehat{\mathbb{G}}_{\mathrm{m}}$ of infinite cyclic groups with index $(n+1) d / \operatorname{gcd}(d, n+1)$. The resulting action of $\mathbb{G}_{\mathrm{m}}$ on $\mathbb{A}(n, r d)$ is defined by the formula $\alpha \cdot f(x)=f\left(\alpha^{-1} x\right)=\alpha^{-r d} f(x)$; thus the index of the subgroup generated by the class of $\Delta$ in $\mathrm{GL}_{n+1} / \boldsymbol{\mu}_{d}$ equals the index of the subgroup of the class of $\Delta$ in $\widehat{\mathbb{G}}_{\mathrm{m}}$ for the action described above, multiplied by the rational number $\operatorname{gcd}(d, n+1) /(n+1) d$.

Now, the action of $\mathbb{G}_{\mathrm{m}}$ described above is induced by the standard action of $\mathbb{G}_{\mathrm{m}}$ defined by the usual formula $\alpha \cdot f(x)=\alpha f(x)$ via the morphism $\mathbb{G}_{\mathrm{m}} \rightarrow \mathbb{G}_{\mathrm{m}}$ defined by $\alpha \mapsto \alpha^{-r d}$; hence, the index of the subgroup generated by the class of $\Delta$ in $\widehat{\mathbb{G}}_{\mathrm{m}}$ for the action above is $r d$ times the class of $\Delta$ in $\widehat{\mathbb{G}}_{\mathrm{m}}$ for the standard action. However, the class of $\Delta$ in $\widehat{\mathbb{G}}_{\mathrm{m}}$ for the standard action is the degree of $\Delta$. Putting all this together, we obtain the following.

Lemma 5.4. If $k$ is a field, the Picard group of the stack $\mathcal{H}_{\mathrm{sm}}(n, r, d)_{k}$ is cyclic of order equal to the degree of the hypersurface $\Delta$ in $\mathbb{A}(n, r d)_{k}$ consisting of singular forms, multiplied by $r \operatorname{gcd}(d, n+$ $1) /(n+1)$.

The hypersurface $\Delta$ in $\mathbb{A}(n, r d)$ is well known to be defined by a polynomial of degree $(n+$ $1)(r d-1)^{n}$ (see for instance [GZK94]); the result would follow if we showed that this polynomial is irreducible when the characteristic of $k$ does not divide $2 r d$. 
Since $\Delta$ is a cone, we can compute its degree as the degree of its projectivization $\bar{\Delta} \subseteq \mathbb{P}(n, r d)$ (recall that $\mathbb{P}(n, r d)$ is the projective space of lines in $\mathbb{A}(n, r d))$. Call $N$ the dimension of $\mathbb{P}(n, r d)$. Let us represent a point of $\mathbb{P}^{n} \times \mathbb{P}(n, r d)$ as a pair $(x, F)$ and let us denote by $D$ the subscheme of $\mathbb{P}^{n} \times \mathbb{P}(n, r d)$ defined by the homogeneous equations $\partial F / \partial x_{i}=0$ with $i=0, \ldots, i=n$; these are $n+1$ equations of bidegree $(r d-1,1)$. The projection $D \rightarrow \mathbb{P}^{n}$ makes $D$ into a $\mathbb{P}^{N-n-1}$ bundle onto $\mathbb{P}^{n}$, hence $D$ is smooth of codimension $n+1$ and a complete intersection. Call $\xi$ and $\eta$ the classes in $\mathrm{A}^{1}\left(\mathbb{P}^{n} \times \mathbb{P}(n, r d)\right)$ obtained by pulling back a hyperplane from $\mathbb{P}^{n}$ and from $\mathbb{P}(n, r d)$, respectively, then the class of $D$ in the Chow ring of $\mathbb{P}^{n} \times \mathbb{P}(n, r d)$ is $((r d-1) \xi+\eta)^{n+1}$; a straightforward calculation, applying projection formula, reveals that its pushforward to $\mathrm{A}^{1}(\mathbb{P}(n, r d))$ has degree $(n+1)(r d-1)^{n}$.

Due to Euler's formula and because the degree $r d$ of a form in $\mathbb{A}(n, r d)$ is not divisible by the characteristic of $k$, if $(x, F)$ is a point of $D$, then $x$ is a singular point of the hypersurface defined by $F$; hence, the image of $D$ in $\mathbb{P}(n, r d)$ is the projectivization $\bar{\Delta}$ of $\Delta$; hence, to conclude the proof it is enough to show that $D$ is birational onto $\bar{\Delta}$. Call $D_{0}$ the inverse image of $D$ in $\mathbb{A}_{0}(n, r d)$; it is enough to show that $D_{0}$ is birational onto its image in $\mathbb{A}_{0}(n, r d)$. We may also assume that the base field $k$ is infinite. It is enough to show that there exists a polynomial $F$ in $\mathbb{A}_{0}(n, r d)(k)$, whose inverse image in $D_{0}$ is a single rational point with the reduced scheme structure. Because of the definition of $F$, this is equivalent to saying that $F$ has a single singular point $p \in \mathbb{P}^{n}(k)$ and the ideal generated by the partial derivates $\partial F / \partial x_{i}$ is the homogeneous ideal of $p$.

Take a polynomial $f \in k[x]$ in one variable of degree $r d$ that has a double root in zero and no other multiple root. We set $f=\sum_{i=1}^{n} a_{i} f\left(x_{i}\right)$, where $a_{1}, \ldots, a_{n}$ are generic elements of $k$ and we call $F$ the homogeneous polynomial of degree $r d$ whose dehomogenization is $f$. We claim that $F$ has the desired property. We immediately check that $F$ has no singularity along the hyperplane at infinity. Furthermore, if $\xi=\left(\xi_{1}, \ldots, \xi_{n}\right)$ is a singular point of $f$, then $0=\partial f / \partial x_{i}(\xi)=a_{i} f^{\prime}\left(\xi_{i}\right)$, so $\xi_{i}$ is a zero of the derivative $f^{\prime}$ of $f$. There are only finitely many such zeros; hence, since the $a_{i}$ are generic $f$ will not vanish on any $n$-tuple $\left(\xi_{1}, \ldots, \xi_{n}\right)$ where each $\xi_{i}$ is a zero of $f^{\prime}$ and at least one of them is different from zero. So the only singularity of $f$ is at the origin. However, $\partial f / \partial x_{i}$ has the form $c_{i} x_{i}+$ higher order terms with all $c_{i}$ different from zero, so the partial derivatives $\partial f / \partial x_{i}$ generate the ideal $\left(x_{1}, \ldots, x_{n}\right) \subseteq k\left[x_{1}, \ldots, x_{n}\right]$. Again by Euler's formula this implies that the ideal generated by the partial derivatives $\partial F / \partial x_{i}$ for $i=0, \ldots, n$ is the homogeneous ideal $\left(x_{1}-x_{0}, \ldots, x_{n}-x_{0}\right)$ and this completes the proof of Theorem 5.1.

Remark 5.5. In particular, this states that the Picard group of the stack of hyperelliptic curves $\mathcal{H}_{\mathrm{sm}}(1,2, g+1)_{k}$ over a field $k$ of characteristic not dividing two or $g+1$ is cyclic of order $2(2 g+1)$ if $g$ is even and $4(2 g+1)$ if $g$ is odd.

When $g=1$, we get that the Picard group of $\mathcal{H}_{\mathrm{sm}}(1,2,1)_{k}$ is cyclic of order 12 ; this immediately reminds us of the famous result of Mumford in [Mum65] that the Picard group of the stack $\mathcal{M}_{1,1}$ is cyclic of order 12. However, as we observed in Example 3.5, part b, $\mathcal{H}_{\mathrm{sm}}(1,2,1)_{k}$ is not isomorphic to $\mathcal{M}_{1,1}$. There is a canonical morphism $\mathcal{M}_{1,1} \rightarrow \mathcal{H}_{\text {sm }}(1,2,1)$, sending a family $\pi: E \rightarrow S$ to the uniform covering $E \rightarrow \mathbb{P}\left(\pi_{*} \mathcal{O}_{E}(2 \Sigma)\right)$, where $\Sigma$ is the image of the given section $S \rightarrow E$; a generator of $\boldsymbol{\mu}_{2}$ acts like the involution $e \mapsto-e$ on $E$. This morphism induced a factorization $\mathcal{M}_{1,1} \rightarrow \mathcal{H}_{\mathrm{sm}}(1,2,1)_{k} \rightarrow \mathcal{M}_{1}$ of the morphism $\mathcal{M}_{1,1} \rightarrow \mathcal{M}_{1}$ forgetting the section.

We claim that this morphism, although it is not an isomorphism, induces an isomorphism of Picard groups. This can be seen as follows. The Picard group of $\mathcal{M}_{1,1}$ is generated by the first Chern class of the Hodge bundle on $\mathcal{M}_{1,1}$. The Hodge bundle is already defined on the stack $\mathcal{M}_{1}$ of unpointed curves of genus 1 and the morphism $\mathcal{M}_{1,1} \rightarrow \mathcal{M}_{1}$ forgetting the section factors through $\mathcal{H}_{\mathrm{sm}}(1,2,1)_{k}$. Hence, there is an element of the Picard group of $\mathcal{H}_{\mathrm{sm}}(1,2,1)_{k}$ mapping into a generator of the Picard group of $\mathcal{M}_{1,1}$. Since both groups are cyclic of the same order, it follows that the pullback homomorphism is, in fact, an isomorphism. 


\section{A. Arsie And A. Vistoli}

\section{Cyclic triple coverings of $\mathbb{P}^{1}$}

In this section we study the stack of cyclic triple covers of the projective line, with particular regard to the smooth ones. General triple covers have been extensively studied in [Mir85].

Definition 6.1. A cyclic triple cover over a scheme $S$ consists of a morphism of $S$-schemes $f: X \rightarrow$ $P$, together with an action of $\boldsymbol{\mu}_{3}$ over $X$ leaving $f$ invariant, such that the following conditions are satisfied.

a) $P \rightarrow S$ is a conic bundle.

b) The morphism $f$ is flat and finite, and induces an isomorphism $X / \boldsymbol{\mu}_{3} \simeq P$.

c) There exists an open subscheme $V \subseteq P$, which intersects every fiber of $f: X \rightarrow P$, such that the restriction $f^{-1}(V) \rightarrow V$ is a $\boldsymbol{\mu}_{3}$-torsor.

Cyclic triple covers can be described by using an eigensheaf decomposition, as for uniform cyclic covers. Consider the action of $\boldsymbol{\mu}_{3}$ on the locally free sheaf $f_{*} \mathcal{O}_{X}$ on $\mathcal{O}_{P}$; this will split as a sum of locally free sheaves of $\mathcal{O}_{P}$-modules $\mathcal{L}_{0} \oplus \mathcal{L}_{1} \oplus \mathcal{L}_{2}$, where $\mathcal{L}_{i}$ is the subsheaf of $f_{*} \mathcal{O}_{X}$ of sections $s$ such that the action of $\boldsymbol{\mu}_{3}$ can be described as $(t, s) \mapsto t^{i} s$. Definition 6.1, condition b, ensures that $\mathcal{L}_{0}=\mathcal{O}_{P}$, while flatness and condition c imply that $\mathcal{L}_{1}$ and $\mathcal{L}_{2}$ are invertible sheaves. The algebra structure on $f_{*} \mathcal{O}_{X}$ induces homomorphisms of sheaves of $\mathcal{O}_{P}$-algebras

$$
\phi_{1}: \mathcal{L}_{1}^{\otimes 2} \longrightarrow \mathcal{L}_{2}, \quad \phi_{2}: \mathcal{L}_{2}^{\otimes 2} \longrightarrow \mathcal{L}_{1} \quad \text { and } \quad \phi_{12}: \mathcal{L}_{1} \otimes \mathcal{L}_{2} \rightarrow \mathcal{O}_{P}
$$

that are injective on every fiber of $P \rightarrow S$. These homomorphisms determine the algebra structure completely; the covering is uniform if and only if $\phi_{1}$ is an isomorphism. The algebra structure also gives homomorphisms $\mathcal{L}_{1}^{\otimes 3} \rightarrow \mathcal{O}_{P}$ and $\mathcal{L}_{2} \rightarrow \mathcal{O}_{P}$ which are injective on every fiber of $P \rightarrow S$ : this states that the degrees of $\mathcal{L}_{i}$ on each fiber of $P \rightarrow S$ cannot be positive. We assume that these degrees are constant on $S$ and we call their opposites $d_{1}$ and $d_{2}$ the branch degrees of the triple covering. These branch degrees are subject to the obvious constraints $0 \leqslant d_{1} \leqslant 2 d_{2}, 0 \leqslant d_{2} \leqslant 2 d_{1}$.

The stack of cyclic triple covers with branch degrees $d_{1}$ and $d_{2}$ will be denoted by $\mathcal{H}\left(1,3 ; d_{1}, d_{2}\right)$; we have $\mathcal{H}(1,3, d)=\mathcal{H}(1,3 ; d, 2 d)$. We denote by $\mathcal{H}_{\mathrm{sm}}\left(1,3 ; d_{1}, d_{2}\right)$ the full subcategory of $\mathcal{H}(1,3 ;$ $\left.d_{1}, d_{2}\right)$ whose objects are triple cyclic covers $X \rightarrow P \rightarrow S$ such that $X$ is smooth over $S$.

Of course all the definitions above generalize to higher dimensions, and we could consider categories of cyclic triple covers of $\mathbb{P}^{n}$ for any $n$; the main reason why we do not do this is that such a cover will never be smooth, unless $n=1$, or the cover becomes uniform after twisting the action by an automorphism of $\boldsymbol{\mu}_{3}$ (see Remark 6.4).

There is an alternate description of $\mathcal{H}\left(1,3 ; d_{1}, d_{2}\right)$. We call $\mathcal{H}^{\prime}\left(1,3 ; d_{1}, d_{2}\right)$ the category whose objects are quintuples $\left(P \rightarrow S, \mathcal{L}_{1}, \mathcal{L}_{2}, \phi_{1}, \phi_{2}\right)$, where $P \rightarrow S$ is a Brauer-Severi scheme of rank one, $\mathcal{L}_{1}$ and $\mathcal{L}_{2}$ are invertible sheaves on $P$, whose degrees on each fiber of $P \rightarrow S$ are $-d_{1}$ and $-d_{2}$, respectively, while $\phi_{1}: \mathcal{L}_{1}^{\otimes 2} \rightarrow \mathcal{L}_{2}$ and $\phi_{2}: \mathcal{L}_{2}^{\otimes 2} \rightarrow \mathcal{L}_{1}$ are homomorphism of sheaves of $\mathcal{O}_{P}$-modules that are injective on all the fibers of $P \rightarrow S$. The arrows are defined in the obvious way.

The construction above yields a functor $\mathcal{H}\left(1,3 ; d_{1}, d_{2}\right) \rightarrow \mathcal{H}^{\prime}\left(1,3 ; d_{1}, d_{2}\right)$; we claim that this is an equivalence of fibered categories over the category of schemes. This is an easy consequence of the following.

Lemma 6.2. Let $Y$ be a scheme and $\mathcal{L}_{1}, \mathcal{L}_{2}$ be invertible sheaves on $Y$, with homomorphisms $\phi_{1}: \mathcal{L}_{1}^{\otimes 2} \rightarrow \mathcal{L}_{2}$ and $\phi_{2}: \mathcal{L}_{2}^{\otimes 2} \rightarrow \mathcal{L}_{1}$. Then $\phi_{1}$ and $\phi_{2}$ extend to a unique structure of associative and commutative $\mathcal{O}_{Y}$-algebra on the $\mathcal{O}_{Y}$-sheaf $\mathcal{O}_{Y} \oplus \mathcal{L}_{1} \oplus \mathcal{L}_{2}$.

Proof. This is a local statement in the Zariski topology, so we may assume that $\mathcal{L}_{1}$ and $\mathcal{L}_{2}$ have global generators $t_{1}$ and $t_{2}$. The homomorphisms $\phi_{1}$ and $\phi_{2}$ correspond to two sections $f_{1}$ and $f_{2}$ of $\mathcal{O}_{Y}$ with $\phi_{1}\left(t_{1} \otimes t_{1}\right)=f_{1} t_{2}$ and $\phi_{2}\left(t_{2} \otimes t_{2}\right)=f_{2} t_{1}$. Set $\mathcal{A} \stackrel{\text { def }}{=} \mathcal{O}_{Y} \oplus \mathcal{L}_{1} \oplus \mathcal{L}_{2}$; to extend $\phi_{1}$ and 


\section{StACKS OF CYCLIC COVERS OF PROJECTIVE SPACES}

$\phi_{2}$ to a bilinear symmetric product $\mathcal{A} \otimes_{\mathcal{O}_{Y}} \mathcal{A} \rightarrow \mathcal{A}$ with identity one, we need to add the data of a homomorphism $\mathcal{L}_{1} \otimes \mathcal{L}_{2} \rightarrow \mathcal{O}_{Y}$, corresponding to a third section $h$ of $\mathcal{O}$ (the image of $t_{1} \otimes t_{2}$ ). Then a lengthy but straightforward calculation reveals that this product is associative if and only if $h=f_{1} f_{2}$, and this clearly implies the result.

A cyclic triple cover $X \rightarrow P$ has two associated branch divisors in $P$, given by the two homomorphisms $\phi_{1}: \mathcal{L}_{1}^{\otimes 2} \rightarrow \mathcal{L}_{2}$ and $\phi_{2}: \mathcal{L}_{2}^{\otimes 2} \rightarrow \mathcal{L}_{1}$, whose degrees are, respectively, $2 d_{1}-d_{2}$ and $2 d_{2}-d_{1}$. We say that the triple cover is smooth if $X$ is smooth over $S$; we denote by $\mathcal{H}_{\mathrm{sm}}\left(1,3 ; d_{1}, d_{2}\right)$ the open substack of $\mathcal{H}\left(1,3 ; d_{1}, d_{2}\right)$ whose objects are smooth triple covers.

Since $X$ is smooth over $S$, to check smoothness it is enough to check that the geometric fibers are smooth.

Proposition 6.3. A cyclic triple cover $X \rightarrow P$ over a field is smooth if and only its two branch divisors have no multiple points, and are disjoint.

Proof. This follows from Proposition 3.1 of [Par91].

We can also proceed as follows. Choose an open subset $U$ of $P$ with non-vanishing sections $t_{1}$ and $t_{2}$ of $\mathcal{L}_{1}$ and $\mathcal{L}_{2}$, respectively. The $\mathcal{O}_{P}$ algebra $\mathcal{O}_{X}$ is defined over $U$ by the equations $t_{1}^{2}=f_{1} t_{2}$, $t_{2}^{2}=f_{2} t_{1}, t_{1} t_{2}=f_{1} f_{2}$ (see the proof of Lemma 6.2). A straightforward calculation using the Jacobian criterion proves that $X$ is smooth over $S$ if and only if $f_{1}$ and $f_{2}$ have no multiple zero and no common zero.

Remark 6.4. We could build a similar theory for projective spaces of dimension higher than one; then a similar argument would show that a triple cover $X \rightarrow P$ is smooth if and only if its two branch divisors are smooth and do not intersect. However, in rank greater than one, this would mean that one of the two divisors must be empty, so that either $d_{2}=2 d_{1}$ and the triple cover is in fact uniform, or $d_{1}=2 d_{2}$ and the triple cover becomes uniform after twisting the action of $\boldsymbol{\mu}_{3}$ by the non-trivial automorphism of $\boldsymbol{\mu}_{3}$ (see Remark 3.6).

Using this description of $\mathcal{H}\left(1,3 ; d_{1}, d_{2}\right)$ we can prove the following. Consider the embedding $\boldsymbol{\mu}_{d_{1}} \times \boldsymbol{\mu}_{d_{2}} \subseteq \mathbb{G}_{\mathrm{m}} \times \mathrm{GL}_{2}$ as a normal subgroup scheme given by

$$
\left(\alpha_{1}, \alpha_{2}\right) \mapsto\left(\alpha_{2} / \alpha_{1}, \alpha_{1} \mathrm{I}_{2}\right)
$$

call $\Gamma\left(d_{1}, d_{2}\right)$ the quotient.

TheOREM 6.5. $\mathcal{H}\left(1,3 ; d_{1}, d_{2}\right)$ is isomorphic to the quotient stack

$$
\left[\mathbb{A}_{0}\left(1,2 d_{1}-d_{2}\right) \times \mathbb{A}_{0}\left(1,2 d_{2}-d_{1}\right) / \Gamma\left(d_{1}, d_{2}\right)\right]
$$

by the action given by the formula

$$
[\alpha, A] \cdot\left(f_{1}(x), f_{2}(x)\right)=\left(\alpha^{d_{2}} f_{1}\left(A^{-1} x\right), \alpha^{-2 d_{2}} f_{2}\left(A^{-1} x\right)\right) .
$$

Furthermore, $U$ is the open subscheme of $\mathbb{A}_{0}\left(1,3,2 d_{1}-d_{2}\right) \times \mathbb{A}_{0}\left(1,3,2 d_{2}-d_{1}\right)$ consisting of pairs of forms without multiple roots and no common root, then $\mathcal{H}_{\mathrm{sm}}\left(1,3 ; d_{1}, d_{2}\right)$ is isomorphic to the quotient $\left[U / \Gamma\left(d_{1}, d_{2}\right)\right]$.

Proof. We closely follow the strategy of the proof of Theorem 4.1 and use the alternative description of $\mathcal{H}\left(1,3 ; d_{1}, d_{2}\right)$ given by $\mathcal{H}^{\prime}\left(1,3 ; d_{1}, d_{2}\right)$.

Consider the auxiliary fibered category $\widetilde{\mathcal{H}}\left(1,3 ; d_{1}, d_{2}\right)$ : if $S$ is a scheme, an object of $\widetilde{\mathcal{H}}(1,3$; $\left.d_{1}, d_{2}\right)(S)$ is a quintuple $\left(P \rightarrow S, \mathcal{L}_{1}, \mathcal{L}_{2}, \phi_{1}, \phi_{2}\right)$ giving an object of $\mathcal{H}^{\prime}\left(1,3 ; d_{1}, d_{2}\right)(S)$, plus the choice of two isomorphisms over $S$

$$
\lambda_{1}:\left(\mathbb{P}_{S}^{1}, \mathcal{O}\left(-d_{1}\right)\right) \simeq\left(P, \mathcal{L}_{1}\right) \text { and } \quad \lambda_{2}:\left(\mathbb{P}_{S}^{1}, \mathcal{O}\left(-d_{2}\right)\right) \simeq\left(P, \mathcal{L}_{2}\right)
$$




\section{A. Arsie And A. Vistoli}

such that the restriction of $\lambda_{2}^{-1} \circ \lambda_{1}$ to $\mathbb{P}_{S}^{1}$ induces the identity on $\mathbb{P}_{S}^{1}$. The arrows are arrows in $\mathcal{H}\left(1,3 ; d_{1}, d_{2}\right)$ preserving the two isomorphisms $\lambda_{1}$ and $\lambda_{2}$. Objects in the fiber $\widetilde{\mathcal{H}}\left(1,3 ; d_{1}, d_{2}\right)(S)$ have no non-trivial automorphism, so $\widetilde{\mathcal{H}}\left(1,3 ; d_{1}, d_{2}\right)$ is equivalent to a functor.

Let $\left(P \rightarrow S, \mathcal{L}_{1}, \mathcal{L}_{2}, \phi_{1}, \phi_{2}, \lambda_{1}, \lambda_{2}\right)$ be an object of $\widetilde{\mathcal{H}}\left(1,3 ; d_{1}, d_{2}\right)(S)$; consider the composition

$$
\left(\mathbb{P}_{S}^{1}, \mathcal{O}\left(-2 d_{1}\right)\right) \stackrel{\lambda_{1}^{\otimes 2}}{\longrightarrow}\left(P, \mathcal{L}_{1}^{\otimes 2}\right) \stackrel{\phi_{1}}{\longrightarrow}\left(P, \mathcal{L}_{2}\right) \stackrel{\lambda_{2}^{-1}}{\longrightarrow}\left(\mathbb{P}_{S}^{1}, \mathcal{O}\left(-d_{2}\right)\right)
$$

(if $\lambda_{1}$ is given by a pair $\left(\mu_{1}, \rho_{1}\right)$, where $\mu_{1}: P \simeq \mathbb{P}_{S}^{1}$ is an isomorphism of $S$-schemes and $\rho_{1}: \mathcal{O}(-1) \simeq$ $\mu_{1}^{*} \mathcal{O}\left(-d_{1}\right)$ is an isomorphism of sheaves of $\mathcal{O}_{\mathbb{P}_{S}^{1}}$-modules, we write $\lambda_{1}^{\otimes 2}$ for the pair $\left.\left(\mu_{1}, \rho_{1}^{\otimes 2}\right)\right)$. This gives a homomorphism of sheaves $\mathcal{O}\left(-2 d_{1}\right) \rightarrow \mathcal{O}\left(-d_{2}\right)$ lying over the identity of $\mathbb{P}_{S}^{1}$, that does not vanish identically on any fiber and, therefore, an element of $\mathbb{A}_{0}\left(1,2 d_{1}-d_{2}\right)(S)$. Analogously we construct an element of $\mathbb{A}_{0}\left(1,2 d_{2}-d_{1}\right)(S)$; this defines a base-preserving functor

$$
\widetilde{\mathcal{H}}\left(1,3 ; d_{1}, d_{2}\right) \longrightarrow \mathbb{A}_{0}\left(1,2 d_{1}-d_{2}\right) \times \mathbb{A}_{0}\left(1,2 d_{2}-d_{1}\right) .
$$

To define an inverse, we send an object $\left(f_{1}, f_{2}\right)$ of $\mathbb{A}_{0}\left(1,2 d_{1}-d_{2}\right)(S) \times \mathbb{A}_{0}\left(1,2 d_{2}-d_{1}\right)(S)$ into the object

$$
\left(\mathbb{P}_{S}^{1} \rightarrow S, \mathcal{O}\left(-d_{1}\right), \mathcal{O}\left(-d_{2}\right), \phi_{1}, \phi_{2}, \text { id }, \mathrm{id}\right)
$$

where $\phi_{1}: \mathcal{O}\left(-2 d_{1}\right) \rightarrow \mathcal{O}\left(-d_{2}\right)$ and $\phi_{2}: \mathcal{O}\left(-2 d_{2}\right) \rightarrow \mathcal{O}\left(-d_{1}\right)$ are given by multiplication by $f_{1}$ and $f_{2}$, respectively. It is straightforward to check that this gives a quasi-inverse to the functor above, so $\widetilde{\mathcal{H}}\left(1,3 ; d_{1}, d_{2}\right)$ is equivalent to $\mathbb{A}_{0}\left(1,2 d_{1}-d_{2}\right) \times \mathbb{A}_{0}\left(1,2 d_{2}-d_{1}\right)$.

Consider the functor $\underline{\mathrm{Aut}}\left(\mathbb{P}_{\mathbb{Z}}^{1}, \mathcal{O}\left(-d_{1}\right), \mathcal{O}\left(-d_{2}\right)\right)$ from schemes to groups, sending a scheme $S$ into the group of automorphisms of the triple $\left(\mathbb{P}_{S}^{1}, \mathcal{O}\left(-d_{1}\right), \mathcal{O}\left(-d_{2}\right)\right)$ over the identity on $S$. This is a sheaf in the fppf topology. It can also be thought of as the fiber product

$$
\underline{\operatorname{Aut}}\left(\mathbb{P}_{\mathbb{Z}}^{1}, \mathcal{O}\left(-d_{1}\right)\right) \times_{\underline{\text { Aut }} \mathbb{P}_{\mathbb{Z}}^{1}} \underline{\operatorname{Aut}}\left(\mathbb{P}_{\mathbb{Z}}^{1}, \mathcal{O}\left(-d_{2}\right)\right) .
$$

Since $\underline{\text { Aut }} \mathbb{P}_{\mathbb{Z}}^{1}$ is $\mathrm{PGL}_{2, \mathbb{Z}}$, and, according to the discussion in the proof of Theorem $4.1, \underline{\text { Aut }}\left(\mathbb{P}_{\mathbb{Z}}^{1}, \mathcal{O}(-d)\right)$ is isomorphic to the quotient $\mathrm{GL}_{2, \mathbb{Z}} / \boldsymbol{\mu}_{d, \mathbb{Z}}$, we see that we have an isomorphism of functors

$$
\underline{\operatorname{Aut}}\left(\mathbb{P}_{\mathbb{Z}}^{1}, \mathcal{O}\left(-d_{1}\right), \mathcal{O}\left(-d_{2}\right)\right) \simeq \mathrm{GL}_{2} \times \mathrm{PGL}_{2} \mathrm{GL}_{2} / \boldsymbol{\mu}_{d_{1}} \times \boldsymbol{\mu}_{d_{2}}
$$

where $\boldsymbol{\mu}_{d_{1}}$ is embedded diagonally in the first copy of $\mathrm{GL}_{2}, \boldsymbol{\mu}_{d_{2}}$ in the second. We also have an isomorphism $\mathbb{G}_{\mathrm{m}} \times \mathrm{GL}_{2} \simeq \mathrm{GL}_{2} \times \mathrm{PGL}_{2} \mathrm{GL}_{2}$, where a section $(\alpha, A)$ of $\mathbb{G}_{\mathrm{m}} \times \mathrm{GL}_{2}$ over some scheme is sent into the pair $(A, \alpha A)$. The embedding $\boldsymbol{\mu}_{d_{1}} \times \boldsymbol{\mu}_{d_{2}} \subseteq \mathrm{GL}_{2} \times \mathrm{PGL}_{2} \mathrm{GL}_{2}$ gives an embedding $\boldsymbol{\mu}_{d_{1}} \times \boldsymbol{\mu}_{d_{2}} \subseteq \mathbb{G}_{\mathrm{m}} \times \mathrm{GL}_{2}$ given by the formula

$$
\left(\alpha_{1}, \alpha_{2}\right) \mapsto\left(\alpha_{2} / \alpha_{1}, \alpha_{1} \mathrm{I}\right) ;
$$

in this way we obtain an isomorphism of $\underline{\operatorname{Aut}}\left(\mathbb{P}_{\mathbb{Z}}^{1}, \mathcal{O}\left(-d_{1}\right), \mathcal{O}\left(-d_{2}\right)\right)$ with $\Gamma\left(d_{1}, d_{2}\right)$.

There is a left action of $\underline{\operatorname{Aut}}\left(\mathbb{P}_{\mathbb{Z}}^{1}, \mathcal{O}\left(-d_{1}\right), \mathcal{O}\left(-d_{2}\right)\right)$ on $\widetilde{\mathcal{H}}\left(1,3 ; d_{1}, d_{2}\right)$; if $\left(P \rightarrow S, \mathcal{L}_{1}, \mathcal{L}_{2}, \phi_{1}, \phi_{2}\right.$, $\left.\lambda_{1}, \lambda_{2}\right)$ is an object of $\widetilde{\mathcal{H}}\left(1,3 ; d_{1}, d_{2}\right)(S)$ and $\left(\alpha_{1}, \alpha_{2}\right)$ is an object of $\underline{\operatorname{Aut}}\left(\mathbb{P}_{\mathbb{Z}}^{1}, \mathcal{O}\left(-d_{1}\right), \mathcal{O}\left(-d_{2}\right)\right)(S)$, we set

$$
\left(\alpha_{1}, \alpha_{2}\right) \cdot\left(P \rightarrow S, \mathcal{L}_{1}, \mathcal{L}_{2}, \phi_{1}, \phi_{2}, \lambda_{1}, \lambda_{2}\right)=\left(P \rightarrow S, \mathcal{L}_{1}, \mathcal{L}_{2}, \phi_{1}, \phi_{2}, \lambda_{1} \circ \alpha_{1}^{-1}, \lambda_{2} \circ \alpha_{2}^{-1}\right) .
$$

Furthermore, given two invertible sheaves $\mathcal{L}_{1}$ and $\mathcal{L}_{2}$ on $P \rightarrow S$ with degrees $-d_{1}$ and $-d_{2}$ on every geometric fiber, there is an fppf covering $S^{\prime} \rightarrow S$, such that the pullback of the triple $\left(P, \mathcal{L}_{1}, \mathcal{L}_{2}\right)$ to $S^{\prime}$ is isomorphic to $\left(\mathbb{P}_{S^{\prime}}^{1}, \mathcal{O}\left(-d_{1}\right), \mathcal{O}\left(-d_{2}\right)\right)$; this fact, plus descent theory, implies that the forgetful morphism $\widetilde{\mathcal{H}}\left(1,3 ; d_{1}, d_{2}\right) \rightarrow \mathcal{H}\left(1,3 ; d_{1}, d_{2}\right)$ makes $\widetilde{\mathcal{H}}\left(1,3 ; d_{1}, d_{2}\right)$ into a principal bundle with structure $\operatorname{group} \underline{\operatorname{Aut}}\left(\mathbb{P}_{\mathbb{Z}}^{1}, \mathcal{O}\left(-d_{1}\right), \mathcal{O}\left(-d_{2}\right)\right)=\Gamma\left(d_{1}, d_{2}\right)$.

The action of $\Gamma\left(d_{1}, d_{2}\right)$ on $\widetilde{\mathcal{H}}\left(1,3 ; d_{1}, d_{2}\right)$ gives an action of the structure group $\underline{\operatorname{Aut}}\left(\mathbb{P}_{\mathbb{Z}}^{1}, \mathcal{O}\left(-d_{1}\right)\right.$, $\left.\mathcal{O}\left(-d_{2}\right)\right)$ on $\mathbb{A}_{0}\left(1,2 d_{1}-d_{2}\right) \times \mathbb{A}_{0}\left(1,2 d_{2}-d_{1}\right)$, via the equivalence above. Hence, $\mathcal{H}\left(1,3 ; d_{1}, d_{2}\right)$ is 


\section{StaCks of CYCLIC COVERS OF PROJECTIVE SPACES}

equivalent to the quotient stack

$$
\left[\mathbb{A}_{0}\left(1,2 d_{1}-d_{2}\right) \times \mathbb{A}_{0}\left(1,2 d_{2}-d_{1}\right) / \Gamma\left(d_{1}, d_{2}\right)\right] ;
$$

now we only have to write this action explicitly.

First of all, restrict attention to the first component $\mathbb{A}_{0}\left(1,2 d_{1}-d_{2}\right)$ and consider the action of $\mathrm{GL}_{2} \times{ }_{\mathrm{PGL}_{2}} \mathrm{GL}_{2}$ on $\mathrm{H}^{0}\left(\mathcal{O}\left(2 d_{1}-d_{2}\right)\right)$. Fix a section $h_{1}$ in $\mathrm{H}^{0}\left(\mathbb{P}_{\mathbb{Z}}^{1}, \mathcal{O}\left(d_{2}\right)\right)$ that does not vanish on any fiber of $\mathbb{P}_{\mathbb{Z}}^{1} \rightarrow$ Spec $\mathbb{Z}$ : any element $f_{1} \in \mathrm{H}^{0}\left(\mathcal{O}\left(2 d_{1}-d_{2}\right)\right)$ can be written uniquely as $f_{1}(x)=g_{1}(x) / h_{1}(x)$, where $g_{1} \in \mathrm{H}^{0}\left(\mathcal{O}\left(2 d_{1}\right)\right)$.

Since the multiplication map $\mathrm{H}^{0}\left(\mathcal{O}\left(2 d_{1}-d_{2}\right)\right) \times \mathrm{H}^{0}\left(\mathcal{O}\left(d_{2}\right)\right) \rightarrow \mathrm{H}^{0}\left(\mathcal{O}\left(2 d_{1}\right)\right)$ is $\mathrm{GL}_{2} \times \mathrm{PGL}_{2} \mathrm{GL}_{2}-$ equivariant, it follows immediately that the action of the group $\mathrm{GL}_{2} \times \mathrm{PGL}_{2} \mathrm{GL}_{2}$ on $H^{0}\left(\mathcal{O}\left(2 d_{1}-d_{2}\right)\right)$ is described by the formula

$$
\left(A_{1}, A_{2}\right) f_{1}(x)=g_{1}\left(A_{1}^{-1} x\right) / h_{1}\left(A_{2}^{-1} x\right) .
$$

Under the isomorphism $\mathbb{G}_{\mathrm{m}} \times \mathrm{GL}_{2} \rightarrow \mathrm{GL}_{2} \times \mathrm{PGL}_{2} \mathrm{GL}_{2}$, given by $(\alpha, A) \mapsto(A, \alpha A)$, the action of $\mathbb{G}_{\mathrm{m}} \times \mathrm{GL}_{2}$ on $H^{0}\left(\mathcal{O}\left(2 d_{1}-d_{2}\right)\right)$ can be written as

$$
(\alpha, A) f_{1}(x)=g_{1}\left(A^{-1} x\right) / h_{1}\left(\alpha^{-1} A^{-1} x\right)=\alpha^{d_{2}} f_{1}\left(A^{-1}(x)\right) .
$$

It is easy to check that this action descends to the quotient group $\Gamma\left(d_{1}, d_{2}\right)$. Analogously, we show that the action of $\Gamma\left(d_{1}, d_{2}\right)$ on the second component $\mathbb{A}_{0}\left(1,2 d_{2}-d_{1}\right)$ is given by the formula

$$
(\alpha, A) f_{2}(x)=\alpha^{-2 d_{2}} f_{2}\left(A^{-1}(x)\right) .
$$

This completes the proof of the first statement.

The last statement is an easy consequence of the definition of the stack of smooth triple covers $\mathcal{H}_{\mathrm{sm}}\left(1,3 ; d_{1}, d_{2}\right)$ and of Proposition 6.3.

In particular, the stack $\mathcal{H}\left(1,3 ; d_{1}, d_{2}\right)$ is a smooth irreducible Artin stack over Spec $\mathbb{Z}$ of dimension $\left(2 d_{1}-d_{2}+1\right)\left(2 d_{2}-d_{1}+1\right)-5$.

Now we give a presentation of the Picard group of the stack $\mathcal{H}_{\mathrm{sm}}\left(1,3 ; d_{1}, d_{2}\right)$.

Theorem 6.6. Assume that $d_{1}$ and $d_{2}$ are positive. Let $R$ be a unique factorization domain, such that the characteristic of its quotient field does not divide $2\left(2 d_{1}-d_{2}\right)\left(2 d_{2}-d_{1}\right)$. Then the Picard group $\operatorname{Pic}\left(\mathcal{H}_{\mathrm{sm}}\left(1,3 ; d_{1}, d_{2}\right)_{R}\right)$ is a group with two generators $v_{1}$ and $v_{2}$ and three relations.

a) If $d_{1}$ is odd, the three relations are

$$
\begin{gathered}
\left(2 d_{1}-d_{2}-1\right)\left(2 v_{1}-\left(d_{2}+2\right) v_{2}\right), \\
\left(2 d_{2}-d_{1}-1\right)\left(4 v_{1}-\left(2 d_{2}+1\right) v_{2}\right), \\
\left(-5 d_{1}+4 d_{2}\right) v_{1}+\frac{4 d_{1}-5 d_{2}\left(d_{1}+1\right)-4 d_{2}^{2}}{2} v_{2} .
\end{gathered}
$$

b) If $d_{1}$ and $d_{2}$ are both even, the three relations are

$$
\begin{gathered}
2\left(2 d_{1}-d_{2}-1\right)\left(v_{1}-2 v_{2}\right), \\
2\left(2 d_{2}-d_{1}-1\right)\left(2 v_{1}-v_{2}\right), \\
\left(4 d_{2}-5 d_{1}\right) v_{1}+\left(4 d_{2}-5 d_{2}\right) v_{2} .
\end{gathered}
$$

Remark 6.7. As is immediately seen, twisting the action by the non-trivial automorphism $\boldsymbol{\mu}_{3} \simeq \boldsymbol{\mu}_{3}$ gives a canonical isomorphism of stacks $\mathcal{H}\left(1,3 ; d_{1}, d_{2}\right) \simeq \mathcal{H}\left(1,3 ; d_{2}, d_{1}\right)$; hence the theorem above describes the Picard group of $\mathcal{H}\left(1,3 ; d_{1}, d_{2}\right)$ even when $d_{1}$ is even and $d_{2}$ is odd.

Proof. The proof is very similar to the proof of Theorem 5.1, with some added complications. Again, Lemma 5.2 allows us to reduce to the case that $R$ is a field. 
A. Arsie And A. Vistoli

The Picard group $\operatorname{Pic}\left(\mathcal{H}_{\mathrm{sm}}\left(1,3 ; d_{1}, d_{2}\right)\right)$ is isomorphic to the codimension one component $\mathrm{A}_{\Gamma\left(d_{1}, d_{2}\right)}^{1}(U)$ of the equivariant Chow ring of the open subscheme $U$ of $\mathbb{A}_{0}\left(1,2 d_{1}-d_{2}\right) \times \mathbb{A}_{0}\left(1,2 d_{2}-d_{1}\right)$ consisting of the complement of three hypersurfaces $\Delta_{1}, \Delta_{2}$ and $Z$ : the first two are the inverse images of the discriminant hypersurfaces of $\mathbb{A}\left(1,2 d_{1}-d_{2}\right)$ and $\mathbb{A}\left(1,2 d_{2}-d_{1}\right)$, respectively, while the geometric points of the third consist of pairs of forms with a common zero (again, this description of $U$ comes from Proposition 6.3). As in the proof of Theorem 5.1, this means that $\mathrm{A}_{\Gamma\left(d_{1}, d_{2}\right)}^{1}(U)$ is the quotient of

$$
\begin{aligned}
\mathrm{A}_{\Gamma\left(d_{1}, d_{2}\right)}^{1}\left(\mathbb{A}_{0}\left(1,2 d_{1}-d_{2}\right) \times \mathbb{A}_{0}\left(1,2 d_{2}-d_{1}\right)\right) & =\mathrm{A}_{\Gamma\left(d_{1}, d_{2}\right)}^{1}(\operatorname{Spec} k) \\
& =\Gamma\left(d_{1}, d_{2}\right)
\end{aligned}
$$

by the subgroups generated by the classes of the three hypersurfaces. The character group $\Gamma \widehat{\left(d_{1}, d_{2}\right)}$ is the kernel of the restriction homomorphism

$$
\widehat{\mathbb{G}_{\mathrm{m}} \times \mathrm{G}} \mathrm{L}_{2} \longrightarrow \widehat{\boldsymbol{\mu}_{d_{1}} \times \boldsymbol{\mu}_{d_{2}}}
$$

The character group of $\mathbb{G}_{\mathrm{m}} \times \mathrm{GL}_{2}$ is generated by the projection $e_{1}: \mathbb{G}_{\mathrm{m}} \times \mathrm{GL}_{2} \rightarrow \mathbb{G}_{\mathrm{m}}$ and by the homomorphism $e_{2}: \mathbb{G}_{\mathrm{m}} \times \mathrm{GL}_{2} \rightarrow \mathbb{G}_{\mathrm{m}}$ defined by $(\alpha, A) \mapsto \operatorname{det} A$. If we denote by $\epsilon_{1}$ and $\epsilon_{2}$ the generators of $\left.\overline{\Gamma\left(d_{1}, d_{2}\right.}\right)$ corresponding to the projection onto $\boldsymbol{\mu}_{d_{1}}$ and $\boldsymbol{\mu}_{d_{2}}$ followed by the embedding into $\mathbb{G}_{\mathrm{m}}$, the restriction homomorphism sends $e_{1}$ into $\epsilon_{2}-\epsilon_{1}$ and $e_{2}$ into $2 \epsilon_{1}$; from this we see that the kernel of the restriction homomorphism is the subgroup of elements of $\mathbb{G}_{\mathrm{m}} \times \mathrm{GL}_{2}$ of the form $x_{1} e_{1}+x_{2} e_{2}$, where $x_{1}$ and $x_{2}$ are integers with $x_{1} \equiv 2 x_{2}\left(\bmod d_{1}\right)$ and $x_{1} \equiv 0\left(\bmod d_{2}\right)$. If $d_{1}$ is odd, then a basis for the kernel is given by

$$
v_{1}=d_{2} e_{1}+\frac{\left(d_{1}+1\right) d_{2}}{2} e_{2} \quad \text { and } \quad v_{2}=d_{1} e_{2}
$$

while if $d_{1}$ and $d_{2}$ are both even a basis is

$$
v_{1}=d_{2} e_{1}+\frac{d_{2}}{2} e_{2} \quad \text { and } \quad v_{2}=\frac{d_{1}}{2} e_{2} .
$$

So the Picard group of $\mathcal{H}\left(1,3 ; d_{1}, d_{2}\right)$ is generated by two elements $v_{1}$ and $v_{2}$, with three relations, obtained by expressing the classes of the three hypersurfaces as linear combinations of $v_{1}$ and $v_{2}$.

To do this we use the following lemma. A cone in $\mathbb{A}\left(2 d_{1}-d_{2}\right)_{k} \times \mathbb{A}\left(2 d_{1}-d_{2}\right)_{k}$ is a closed subscheme that is invariant under the actions of $\mathbb{G}_{\mathrm{m}} \times \mathbb{G}_{\mathrm{m}}$ defined by $\left(t_{1}, t_{2}\right) \cdot\left(f_{1}, f_{2}\right)=\left(t_{1} f_{1}, t_{2} f_{2}\right)$. The integral cones correspond to the integral subschemes of $\mathbb{P}\left(2 d_{1}-d_{2}\right)_{k} \times \mathbb{P}\left(2 d_{1}-d_{2}\right)_{k}$ and, as such, they have a bidegree.

Lemma 6.8. Let $S$ be an integral cone of codimension one in $\mathbb{A}\left(2 d_{1}-d_{2}\right)_{k} \times \mathbb{A}\left(2 d_{1}-d_{2}\right)_{k}$ of bidegree $\left(a_{1}, a_{2}\right)$ that is invariant under the action of $\Gamma\left(d_{1}, d_{2}\right)$.

a) If $d_{1}$ is odd, the integer $4 a_{2} d_{2}-a_{2} d_{1}-a_{1} d_{1} d_{2}$ is divisible by $2 d_{1}$ and the class of $S$ in $\left.\Gamma \widehat{\left(d_{1}, d_{2}\right.}\right)$ is

$$
\left(a_{1}-2 a_{2}\right) v_{1}+\left(-a_{1}+a_{2} d_{2}+a_{2} a_{1} d_{2} / 2\right) v_{2} .
$$

b) If $d_{1}$ and $d_{2}$ are both even, the integer $4 a_{2} d_{2}$ is divisible by $d_{1}$ and the class of $S$ in $\Gamma \widehat{\left(d_{1}, d_{2}\right)}$ is

$$
\left(a_{1}-2 a_{2}\right) v_{1}+\left(-2 a_{1}+a_{2}\right) v_{2} .
$$

Proof. Let $\Phi$ be a generator of the ideal of $S$. Saying that $S$ has bidegree $\left(a_{1}, a_{2}\right)$ is the same as saying that $\Phi\left(t_{1} f_{1}, t_{2} f_{2}\right)=t_{1}^{a_{1}} t_{2}^{a_{2}} \Phi\left(f_{1}, f_{2}\right)$ for any $\left(t_{1}, t_{2}\right)$ in $\mathbb{G}_{\mathrm{m}} \times \mathbb{G}_{\mathrm{m}}$ and any $\left(f_{1}, f_{2}\right)$ in $\mathbb{A}\left(2 d_{1}-\right.$ $\left.d_{2}\right)_{k} \times \mathbb{A}\left(2 d_{1}-d_{2}\right)_{k}$. On the other hand, since $S$ is also invariant for the action of $\mathbb{G}_{\mathrm{m}} \times \mathrm{GL}_{2}$, we must have a formula of the type

$$
\Phi\left(\alpha^{d_{2}} f_{1}\left(A^{-1} x\right), \alpha^{-2 d_{2}} f_{2}\left(A^{-1} x\right)\right)=\alpha^{n_{1}}(\operatorname{det} A)^{n_{2}} \Phi\left(f_{1}, f_{2}\right) ;
$$




\section{StACKS OF CYCLIC COVERS OF PROJECTIVE SPACES}

furthermore, in this case, the class of $S$ is $n_{1} e_{1}+n_{2} e_{2}$, where $e_{1}$ and $e_{2}$ are generators of $\widehat{\mathbb{G}_{\mathrm{m}} \times \mathrm{G}} \mathrm{L}_{2}$. To compute the integers $n_{1}$ and $n_{2}$ we set $A=\beta \mathrm{I}_{2}$, where $\beta$ is scalar, so that $\operatorname{det} A=\beta^{2}$. We get

$$
\begin{aligned}
\Phi\left(\alpha^{d_{2}} f_{1}\left(\beta^{-1} x\right), \alpha^{-2 d_{2}} f_{2}\left(\beta^{-1} x\right)\right) & =\Phi\left(\alpha^{d_{2}} \beta^{-2 d_{1}+d_{2}} f_{1}(x), \alpha^{-2 d_{2}} \beta^{-2 d_{1}+d_{2}} f_{2}(x)\right) \\
& =\alpha^{\left(a_{1}-2 a_{2}\right) d_{2}} \beta^{-a_{1}\left(2 d_{1}-d_{2}\right)-a_{2}\left(2 d_{2}-d_{1}\right)} \Phi\left(f_{1}, f_{2}\right)
\end{aligned}
$$

hence

$$
\Phi\left(\alpha^{d_{2}} f_{1}\left(A^{-1} x\right), \alpha^{-2 d_{2}} f_{2}\left(A^{-1} x\right)\right)=\alpha^{\left(a_{1}-2 a_{2}\right) d_{2}}(\operatorname{det} A)^{-\left(a_{1}\left(2 d_{1}-d_{2}\right)+a_{2}\left(2 d_{2}-d_{1}\right)\right) / 2} \Phi\left(f_{1}, f_{2}\right)
$$

and from this we obtain that the class of $S$ in $\widehat{\mathbb{G}_{\mathrm{m}} \times \mathrm{G}} \mathrm{L}_{2} \simeq \mathbb{Z}^{2}$ is

$$
\left(a_{1}-2 a_{2}\right) d_{2} e_{1}-\frac{a_{1}\left(2 d_{1}-d_{2}\right)+a_{2}\left(2 d_{2}-d_{1}\right)}{2} e_{2} .
$$

The result follows by expressing this class as a linear combination of $v_{1}$ and $v_{2}$.

This reduces the problem to computing the bidegrees of the three hypersurfaces. The hypersurface $\Delta_{1}$ is the pullback of the discriminant hypersurface from the first factor $\mathbb{A}\left(2 d_{1}-d_{2}\right)$ and we have seen in the proof of Theorem 5.1 that this is integral with degree $2\left(2 d_{1}-d_{2}-1\right)$. Hence the bidegree of $\Delta_{1}$ is $\left(2\left(2 d_{1}-d_{2}-1\right), 0\right)$ and, if we plug this in the formulas of Lemma 6.8, we obtain that the class of $\Delta_{1}$ is

$$
2\left(2 d_{1}-d_{2}-1\right) v_{1}-\left(d_{2}+2\right)\left(2 d_{1}+d_{2}-1\right) v_{2}
$$

when $d_{1}$ is odd, and

$$
2\left(2 d_{1}-d_{2}-1\right) v_{1}-4\left(2 d_{1}-d_{2}-1\right) v_{2}
$$

when $d_{1}$ and $d_{2}$ are both even. This gives us our first relation.

The second is obtained similarly, by setting $a_{1}=0$ and $a_{2}=2\left(2 d_{2}-d_{1}-1\right)$ in the formulas; the result is

$$
2\left(2 d_{1}-d_{2}-1\right) v_{1}-4\left(2 d_{1}-d_{2}-1\right) v_{2}
$$

when $d_{1}$ is odd, and

$$
-4\left(2 d_{2}-d_{1}-1\right) v_{1}+\left(2 d_{2}+1\right)\left(2 d_{2}-d_{1}-1\right) v_{2}
$$

when $d_{1}$ and $d_{2}$ are both even.

To calculate the bidegree of $Z$, consider the subscheme $\widetilde{Z}$ of $\mathbb{P}^{1} \times \mathbb{P}\left(2 d_{1}-d_{2}\right) \times \mathbb{P}\left(2 d_{2}-d_{1}\right)$ consisting of triples $\left(p, f_{1}, f_{2}\right)$, where $p$ is a point in $\mathbb{P}^{1}$ and $f_{1}, f_{2}$ are forms vanishing at $p$. Then $\widetilde{Z}$ is a smooth subscheme of codimension two, and the projection

$$
\mathbb{P}^{1} \times \mathbb{P}\left(2 d_{1}-d_{2}\right) \times \mathbb{P}\left(2 d_{2}-d_{1}\right) \longrightarrow \mathbb{P}\left(2 d_{1}-d_{2}\right) \times \mathbb{P}\left(2 d_{2}-d_{1}\right)
$$

maps $\widetilde{Z}$ birationally onto $Z$. If we denote by $\eta, \xi_{1}$ and $\xi_{2}$ the pullbacks to $\mathbb{P}^{1} \times \mathbb{P}\left(2 d_{1}-d_{2}\right) \times \mathbb{P}\left(2 d_{2}-d_{1}\right)$ of the first Chern classes of $\mathcal{O}(1)$ on $\mathbb{P}^{1}, \mathbb{P}\left(2 d_{1}-d_{2}\right)$ and $\mathbb{P}\left(2 d_{2}-d_{1}\right)$, respectively, then the class of $\widetilde{Z}$ in the Chow ring of $\mathbb{P}^{1} \times \mathbb{P}\left(2 d_{1}-d_{2}\right) \times \mathbb{P}\left(2 d_{2}-d_{1}\right)$ is

$$
\left(\left(2 d_{1}-d_{2}\right) \eta+\xi_{1}\right)\left(\left(2 d_{2}-d_{1}\right) \eta+\xi_{2}\right) .
$$

By pushing this class forward to $\mathbb{P}\left(2 d_{1}-d_{2}\right) \times \mathbb{P}\left(2 d_{2}-d_{1}\right)$, using projection formula, we see that the bidegree of $Z$ is $\left(2 d_{2}-d_{1}, 2 d_{1}-d_{2}\right)$. Again we use the formulas of Lemma 6.8 to obtain the third relation

when $d_{1}$ is odd, and

$$
\left(-5 d_{1}+4 d_{2}\right) v_{1}+\frac{4 d_{1}-5 d_{2}\left(d_{1}+1\right)-4 d_{2}^{2}}{2} v_{2}
$$

$$
\left(-5 d_{1}+4 d_{2}\right) v_{2}+\left(4 d_{1}-5 d_{2}\right) v_{2}
$$

when $d_{1}$ and $d_{2}$ are both even. 


\section{StACKS OF CYCLIC COVERS OF PROJECTIVE SPACES}

Remark 6.9. There are two possible generalizations of this theory. Given that general flat covers of $\mathbb{P}^{1}$ seem completely out of reach, we could study the stack of cyclic covers of a conic of degree $r$ for fixed $r$, or the stack of general triple covers.

For general triple covers of conics, we can use the description of [Mir85]. This is a work in progress of Marco Barone, a student of the second author. There is one difficulty: although every locally free sheaf of rank two on $\mathbb{P}_{k}^{1}$ is isomorphic to $\mathcal{O}(m) \oplus \mathcal{O}(n)$, if $k$ is a field, this is not true over an arbitrary base. This means that we can mimics the construction of Theorem 6.5 and use the results of [Mir85] to study the stack of triple coverings $f: X \rightarrow P$ where $P \rightarrow S$ is a conic bundle and the kernel of the trace map $f_{*} \mathcal{O}_{X} \rightarrow \mathcal{O}_{P}$ is assumed to be locally isomorphic to $\mathcal{O}(m) \oplus \mathcal{O}(n)$ for fixed $m$ and $n$. However, removing this unpleasant restriction requires a new idea.

For general cyclic covers (or, more generally, covers that are generically torsors under a finite diagonalizable group) there is the theory created by Pardini (see [Par91]). Using her 'reduced building data' we can describe the stack of all cyclic smooth covers of Brauer-Severi varieties as a quotient of an open subset of a representation of a quotient of a product of general linear groups; but for non-smooth covers, her description does not work in general and only yields a stack that is birational to the stack of cyclic covers.

\section{ACKNOWLEDGEMENTS}

The authors are grateful to Zinovy Reichstein and Rita Pardini for useful discussions.

This paper can be considered as a tribute to the incredible mathematical insight of David Mumford, who wrote [Mum65] almost forty years ago. Reading this paper was a high point in the mathematical education of the second author.

\section{REFERENCES}

Cat84 F. Catanese, On the moduli spaces of surfaces of general type, J. Differential Geom. 19 (1984), $483-515$.

EG98 D. Edidin and W. Graham, Equivariant intersection theory, Invent. Math. 131 (1998), 595-634.

GZK94 I. M. Gelfand, M. M. Kapranov and A. V. Zelevinsky, Discriminants, resultants and multidimensional determinants (Birkhäuser, Boston, 1994).

Kir85 F. Kirwan, Partial desingularisations of quotients of nonsingular varieties and their Betti numbers, Ann. Math. 122 (1985), 41-85.

LM00 G. Laumon and L. Moret-Bailly, Champs algébrique (Springer, 2000).

Mir85 R. Miranda, Triple covers in algebraic geometry, Amer. J. Math. 107 (1985), 113-1158.

Mum65 D. Mumford, Picard groups of moduli problems, in Arithmetical algebraic geometry (Proc. Conf. Purdue Univ., 1963) (Harper \& Row, New York, 1965), 33-81.

Par91 R. Pardini, Abelian covers of algebraic varieties, J. Reine Angew. Math. 417 (1991), 191-213.

Vis98 A. Vistoli, The Chow ring of $\mathcal{M}_{2}$, Invent. Math. 131 (1998), 635-644, an appendix to [EG98].

Alessandro Arsie arsie@dm.unibo.it

Dipartimento di Matematica, Università di Bologna, 40126 Bologna, Italy

Angelo Vistoli vistoli@dm.unibo.it

Dipartimento di Matematica, Università di Bologna, 40126 Bologna, Italy 